\title{
Third and Higher Order NFPA Twisted Constructions of Conformal Field Theories from Lattices
}

\author{
P.S. Montague \\ Department of Applied Mathematics and Theoretical Physics \\ University of Cambridge \\ Silver Street \\ Cambridge CB3 9EW \\ U.K.
}

May 5, 2018

\begin{abstract}
We investigate orbifold constructions of conformal field theories from lattices by no-fixed-point automorphisms (NFPA's) $\mathbf{Z}_{p}$ for $p$ prime, $p>2$, concentrating on the case $p=3$. Explicit expressions are given for most of the relevant vertex operators, and we consider the locality relations necessary for these to define a consistent conformal field theory. A relation to constructions of lattices from codes, analogous to that found in earlier work in the $p=2$ case which led to a generalisation of the triality structure of the Monster module, is also demonstrated.
\end{abstract}

\section{Introduction}

Over the last ten years, conformal field theory has exhibited a massive development, with diverse applications in string theory and two-dimensional critical phenomena as well as more abstract areas of mathematics [6, 33, 0, 20, 18, 19]. We consider, in this paper, an explicit construction of a class of theories with possible applications in many of these different arenas.

In earlier work [15], an explicit general construction was given for $\mathbf{Z}_{2}$-twisted orbifolds of the FKS lattice theories, motivated by the fact that one such twisted theory (that corresponding to the Leech lattice) was shown by Frenkel, Lepowsky and Meurman (FLM) [20] to form a natural module $V^{\natural}$ for the Monster group $\mathbf{M}$. (Note that there are direct string-theoretic interpretations in terms of propagation of bosonic strings on tori and orbifolds thereof.) This construction extended the results of previous work [24, 9, 10] by addressing a normal ordering ambiguity in the twisted sectors, and, more importantly, full consistency of the conformal field theory was demonstrated by verification of all the relevant locality relations. 
FLM had shown that the specific features of $V^{\natural}$ led to the existence of a "triality" involution which, together with the obvious automorphisms acting on the theory obtained by lifting automorphisms of the lattice, generated $\mathbf{M}$. It was shown [16, 14 that this triality could be extended to a larger class of $\mathbf{Z}_{2}$-twisted theories, by relating the straight (FKS) and twisted constructions to analogous constructions of lattices from binary codes, showing that the code structure which had hitherto been thought of as merely providing a (useful) analogy to conformal field theory in fact plays a much more fundamental role in the structure of such theories. In establishing symmetries of conformal field theories and the equivalence of potentially distinct theories, our approach helps provide a tool for a fuller understanding of their structure.

In this paper, we consider $\mathbf{Z}_{3}$-twisted (no-fixed-point) orbifolds of the FKS theories specifically, though $\mathbf{Z}_{p}$-twists for other primes $p$ are similar, and are given brief consideration. New features arise that do not occur in the $\mathbf{Z}_{2}$ case - in particular we lose the advantage of having a completely explicit construction. Some of the expressions derived have been found previously in string-theory related work [21], but our results are more complete, including, for example, discussion of the twisted sector cocycles. The locality relations are considered, and then we go on to describe a pair of constructions of Eisenstein lattices from ternary codes which are found to interconnect with the straight and $\mathbf{Z}_{3}$-twisted conformal field theory constructions from lattices in a similar way to binary codes in the $\mathbf{Z}_{2}$ case. This promises to allow a similar generalisation of an analogue of triality to a wider class of theories, since Dong and Mason have demonstrated that the $\mathbf{Z}_{3}$-twisted Leech lattice theory is again the Monster module $V^{\natural}$ [17], as well as at a more basic level providing tools for the explicit construction of the complete class of self-dual theories at central charge 24 [33, 34, 35].

Also, the detailed analysis given in this paper for such relatively simple examples of twisted conformal field theories will be of use in the explicit calculation of conjectured properties of such models.

The work of the paper can be seen again as extending that of Corrigan and Holowood, who discussed the algebra of vertex operators in twisted conformal field theories under certain assumptions about operator product expansions. We try to take this further and present a systematic approach to provide a more complete definition and remove any need for assumptions by fully exploiting the powerful consequences of locality - the verification of which then forms the bulk of the remaining work.

Much of the physical motivation present for the $\mathbf{Z}_{2}$ case in [15] remains true here. Many of the interesting applications of conformal field theory involve twisted fields [12, 13, 11], perhaps the simplest example being the analysis of critical phenomena in the 2-d Ising model, where the spin field is a map from an untwisted to a twisted sector. In superstring models, the set of space-time fermionic fields is an example of twisted conformal fields, and a complete analysis of the fermion emission vertex in these theories would be the analogue of that provided here for bosonic "spin" operators. Realistic string models also frequently contain complex, e.g. $\mathbf{Z}_{n}$-twisted world sheet fermionic and bosonic fields, described in terms of orbifolds or otherwise [12, 13, [1, 5, 2, 1]. A comprehensive description of these theories, including their explicit construction in terms of local conformal fields, and an understanding of the algebras they form, will undoubtedly be of use in singling out 
interesting physical applications.

\section{Conformal Field Theories}

We begin by defining what shall be meant by a conformal field theory (see [14 for a fuller discussion of the axioms and their consequences). Essentially, we shall deal with vertex operator algebras [6] or, as physicists would categorise them, chiral bosonic meromorphic chiral conformal field theories.

A conformal field theory consists of a Hilbert space $\mathcal{H}$, a set $\mathcal{V}=\{V(\psi, z): \psi \in \mathcal{H}, z \in$ C \} of "vertex operators" (linear maps from $\mathcal{H}$ into itself) and a pair of states $|0\rangle, \psi_{L} \in \mathcal{H}$ such that

- $V(\psi, z) V(\phi, w)=V(\phi, w) V(\psi, z)$, the locality axiom. Note that the left hand side is strictly defined only for $|z|>|w|$, and similarly for the right hand side, and so we are to interpret this equality in the sense that on taking matrix elements of either side the resulting meromorphic functions are analytic continuations of one another. This axiom is physically reasonable in that we are to interpret the vertex operator $V(\psi, z)$ as representing the insertion of the state $\psi$ on to the world sheet of a string at the point $z$. For a bosonic string theory, the order of such operator insertions must clearly be irrelevant.

- $V\left(\psi_{L}, z\right)=\sum_{n} L_{n} z^{-n-2}$, with

$$
\left[L_{m}, L_{n}\right]=(m-n) L_{m+n}+\frac{c}{12} m\left(m^{2}-1\right) \delta_{m,-n},
$$

for some scalar $c$, known as the central charge.

- $V(\psi, z)|0\rangle=e^{z L_{-1}} \psi$, the creation axiom.

The remaining axioms are technicalities, listed here only for the sake of completeness.

- $x^{L_{0}}$ acts locally with respect to $\mathcal{V}$, i.e. $x^{L_{0}} V(\psi, z) x^{-L_{0}}$ is local with respect to all the vertex operators in $\mathcal{V}$.

- The spectrum of $L_{0}$ is bounded below.

- $|0\rangle$ is the only state annihilated by $L_{0}, L_{ \pm 1}$, i.e. the only su(1,1) invariant state in the theory.

If, in addition, the theory is such that

- $V\left(e^{z^{*} L_{1}} z^{*-2 L_{0}} \psi, 1 / z^{*}\right)^{\dagger}$ is local with respect to $\mathcal{V}$, i.e. 115

$$
V\left(e^{z^{*} L_{1}} z^{*-2 L_{0}} \psi, 1 / z^{*}\right)^{\dagger}=V(\bar{\psi}, z)
$$

for some antilinear map $\mathcal{H} \rightarrow \mathcal{H}$,

then we say that it is hermitian. 


\section{The Straight Construction in the $\mathbb{Z}_{3}$ Context}

In this section, we describe the FKS or straight (i.e. untwisted) construction of a conformal field theory from a lattice which admits a third order NFPA. This is simply a reformulation of the construction described, for example, in [15, 24], but it serves to introduce our notation.

\subsection{Lattice Structure}

Firstly, we will discuss a reformulation of the lattice structure behind the construction. Anticipating a connection with ternary codes, we take our lattice to be a $J$-module, for $J$ a ring other than $\mathbf{Z}$, which admits a natural $\mathbf{Z}_{3}$ structure. The obvious choice is the Eisenstein integers $\mathcal{E}=\{m+n \omega: m, n \in \mathbf{Z}\}$, where $\omega=e^{2 \pi i / 3}$, for which multiplication by $\omega$ is a natural $\mathbf{Z}_{3}$ symmetry.

The generalisation of the concept of a lattice to a ring $J$ is given as follows [8]. Let $K$ be the corresponding field to $J$, e.g. $K=\mathbf{R}$ for $J=\mathbf{Z}, K=\mathbf{C}$ for $J=\mathcal{E}$. Let $e_{1}, \ldots e_{d} \in K^{d}$ be linearly independent over $K$. Then the $J$-lattice $\Lambda$ is defined by

$$
\Lambda=\left\{\lambda_{1} e_{1}+\ldots+\lambda_{d} e_{d}: \lambda_{i} \in J, 1 \leq i \leq d\right\}
$$

Since $J$ is a ring, we see that $\Lambda$ is closed under addition, subtraction and multiplication by elements of $J$, i.e. it is a $J$-module. A choice for $J$ which will be of relevance later is $J=\mathbf{Z}\left[\omega_{p}\right]$, the ring of cyclotomic integers, where $\omega_{p}=e^{2 \pi i / p}, p$ prime, i.e. $\mathbf{Z}\left[\omega_{p}\right]=$ $\left\{m_{0}+m_{1} \omega_{p}+\ldots+m_{p-1} \omega_{p}^{p-1}: m_{0}, \ldots, m_{p-1} \in \mathbf{Z}\right\}$, with corresponding field $K=\mathbf{C}$. In particular, $\mathbf{Z}\left[\omega_{3}\right]=\mathcal{E}$. Because of the natural $\mathbf{Z}_{3}$ symmetry of $\mathcal{E}$, an $\mathcal{E}$-lattice $\Lambda$ automatically admits a third order automorphism $\lambda \mapsto \omega \lambda$ (the scalar product on the lattice is taken to be $(\lambda, \mu)=\lambda \cdot \bar{\mu})$.

We may define concepts analogous to those defined for Z Z-lattices. In particular, the dual lattice $\Lambda^{*}=\left\{x \in K^{d}: x \cdot \bar{u} \in J \forall u \in \Lambda\right\} . \Lambda$ is said to be integral if $\Lambda \subset \Lambda^{*}$ and self-dual if $\Lambda=\Lambda^{*}$. It is known that there are no even self-dual $\mathcal{E}$-lattices. (Note that, for $\Lambda$ integral, $\lambda \cdot \bar{\lambda}$ is a real element of $\mathcal{E}$, i.e. $\lambda \cdot \bar{\lambda} \in \mathbf{Z}$, so that this statement does make sense.) This result provides a justification for the claim that an $\mathcal{E}$-lattice is the appropriate analogue of a ternary code from which to begin since there are no self-dual ternary codes whose weights are all multiples of 6 (see e.g. [29]).

We may construct a $2 d$-dimensional $\mathbf{Z}$-lattice $\Lambda_{R}$ from the $d$-dimensional $\mathcal{E}$-lattice $\Lambda$ [8]. Suppose $\Lambda$ has generator matrix $X+i Y$, with $X, Y$ real (i.e. $X+i Y$ is a $d \times d$ complex matrix whose rows $\alpha_{1}, \ldots, \alpha_{d}$ comprise a basis for $\Lambda$ ). Then we can define the lattice $\Lambda_{R}$ by specifying its generator matrix to be

$$
\left(\begin{array}{cc}
\sqrt{2} X & \sqrt{2} Y \\
-\frac{1}{\sqrt{2}}(X+\sqrt{3} Y) & -\frac{1}{\sqrt{2}}(Y-\sqrt{3} X)
\end{array}\right)
$$

with rows $b_{1}, \ldots, b_{2 d}$. 
Define

$$
\begin{aligned}
\langle\lambda, \mu\rangle & =(\lambda, \mu)+(\mu, \lambda) \\
& =(\lambda, \mu)+\overline{(\lambda, \mu)} \\
& =2 \operatorname{Re}(\lambda, \mu),
\end{aligned}
$$

for $\lambda, \mu \in \Lambda .\langle\cdot, \cdot\rangle$ is a real symmetric bilinear form. Clearly $b_{i} \cdot b_{j}=2 \operatorname{Re}\left(\alpha_{i} \cdot \bar{\alpha}_{j}\right)=$ $\left\langle\alpha_{i}, \alpha_{j}\right\rangle=\left\langle\omega \alpha_{i}, \omega \alpha_{j}\right\rangle$ for $1 \leq i, j \leq d$ or $d+1 \leq i, j \leq 2 d$, and for $1 \leq i \leq d$, $d+1 \leq j \leq 2 d b_{i} \cdot b_{j}=-\operatorname{Re}\left(\alpha_{i} \cdot \bar{\alpha}_{j}\right)+\sqrt{3} \operatorname{Im}\left(\alpha_{i} \cdot \bar{\alpha}_{j}\right)=\left\langle\alpha_{i}, \omega \alpha_{j}\right\rangle$. Thus, we have a map $T: \Lambda_{R} \rightarrow \Lambda$ given by

$$
T\left(\sum_{i=1}^{2 d} m_{i} b_{i}\right)=\sum_{i=1}^{d} m_{i} \alpha_{i}+\sum_{i=d+1}^{2 d} m_{i} \omega \alpha_{i-d}
$$

such that

$$
\rho \cdot \gamma=\langle T \rho, T \gamma\rangle
$$

for $\rho, \gamma \in \Lambda_{R}$. Hence, if we restrict $\Lambda$ to be integral, then $\rho \cdot \gamma=2 \operatorname{Re}(T \rho, T \gamma) \in \mathbf{Z}$ since $(\cdot, \cdot): \Lambda_{R} \times \Lambda_{R} \rightarrow \mathcal{E}$, i.e. $\Lambda_{R}$ is integral. Also $\rho^{2}=2 \operatorname{Re}(T \rho, T \rho)$. But $(T \rho, T \rho) \in \mathcal{E}$ and is real, so $(T \rho, T \rho) \in \mathbf{Z}$ and $\rho^{2} \in 2 \mathbf{Z}$. Hence, $\Lambda_{R}$ is in fact even if $\Lambda$ is integral.

\subsection{Hilbert Space Structure and Vertex Operators}

We introduce two sets of oscillators $b_{n}^{i}, \bar{b}_{n}^{i}, 1 \leq i \leq d$, such that

$$
\begin{aligned}
{\left[b_{m}^{i}, \bar{b}_{n}^{j}\right] } & =m \delta_{m,-n} \delta^{i j} \\
{\left[b_{m}^{i}, b_{n}^{j}\right]=} & =\left[\bar{b}_{m}^{i}, \bar{b}_{n}^{j}\right] \\
b_{n}^{i \dagger} & =\bar{b}_{-n}^{i} \\
\left(p \equiv b_{0}^{i}\right)|\lambda\rangle & =\frac{\lambda}{\alpha}|\lambda\rangle, \quad \lambda \in \Lambda \\
\left(\bar{p} \equiv \bar{b}_{0}^{i}\right)|\lambda\rangle & =\frac{\bar{\lambda}}{\alpha}|\lambda\rangle \\
b_{n}^{i}|\lambda\rangle=\bar{b}_{n}^{i}|\lambda\rangle & =0, \quad n>0,
\end{aligned}
$$

for some integral Eisenstein lattice $\Lambda$ and some scale factor $\alpha$, fixed later by considerations of self-duality and locality.

For

$$
\psi=\prod_{a=1}^{M} b_{-m_{a}}^{i_{a}} \prod_{b=1}^{N} \bar{b}_{-n_{b}}^{j_{b}}|\lambda\rangle
$$

we have [see e.g. [24]]

$$
V(\psi, z)=: \prod_{a=1}^{M} \frac{i}{\left(m_{a}-1\right) !} \frac{d^{m_{a}}}{d z^{m_{a}}} X_{+}^{i_{a}}(z) \prod_{b=1}^{N} \frac{i}{\left(n_{b}-1\right) !} \frac{d^{n_{b}}}{d z^{n_{b}}} X_{-}^{j_{b}}(z) e^{i \frac{\bar{\lambda}}{\alpha} \cdot X_{+}(z)} e^{i \frac{\lambda}{\alpha} \cdot X_{-}(z)}: \sigma_{\lambda},
$$


where

$$
X_{+}(z)=q-i p \ln z+i \sum_{n \neq 0} \frac{b_{n}}{n} z^{-n}
$$

and

$$
X_{-}(z)=\bar{q}-i \bar{p} \ln z+i \sum_{n \neq 0} \frac{\bar{b}_{n}}{n} z^{-n}
$$

with

$$
e^{i \frac{\bar{\lambda}}{\alpha} \cdot q+(z)} e^{i \frac{\lambda}{\alpha} \cdot \bar{q}}|\mu\rangle=|\lambda+\mu\rangle
$$

The relation

$$
\hat{\sigma}_{\lambda}\left(\equiv e^{i \frac{\bar{\lambda}}{\alpha} \cdot q+(z)} e^{i \frac{\lambda}{\alpha} \cdot \bar{q}}\right) \hat{\sigma}_{\mu}=(-1)^{\langle\lambda, \mu\rangle / \alpha^{2}} \hat{\sigma}_{\mu} \hat{\sigma}_{\lambda}
$$

ensures locality [15].

The third order automorphism $\theta$ in this picture is simply given by

$$
\begin{aligned}
\theta b_{n}^{i} \theta^{-1} & =\omega b_{n}^{i} \\
\theta \bar{b}_{n}^{i} \theta^{-1} & =\bar{\omega} \bar{b}_{n}^{i} \\
\theta|\lambda\rangle & =|\bar{\omega} \lambda\rangle
\end{aligned}
$$

(It is easily checked that this is an automorphism, provided that $\theta \sigma_{\lambda} \theta^{-1}=\sigma_{\bar{\omega} \lambda}$. See [15] for a discussion of the representation of the cocycle operators.) The preferred states $|0\rangle$ and $\psi_{L}$ are given respectively by the vacuum state $|0\rangle$ and $b_{-1} \cdot \bar{b}_{-1}|0\rangle$. The moments of $V\left(\psi_{L}, z\right)$ are then found to be

$$
L_{n}=: \sum_{m=-\infty}^{\infty} b_{m} \cdot \bar{b}_{n-m}:
$$

and also to satisfy the Virasoro algebra with $c=2 d$. The remaining axioms are easily checked. Note that the conformal weight of the state (11) is given by

$$
h_{\psi}=\frac{(\lambda, \lambda)}{\alpha^{2}}+\sum_{a=1}^{M} m_{a}+\sum_{b=1}^{N} n_{b} .
$$

Thus, we see that the theory is meromorphic provided $(\lambda, \lambda) \in \alpha^{2} \mathbf{Z}$ for all $\lambda \in \Lambda$. If we impose this condition on the lattice $\Lambda$, then the theory is just the standard FKS construction on the even $\left(\mathbf{Z}\right.$-)lattice $\Lambda_{R} / \alpha$.

Finally, note that there exists a hermitian structure, with

$$
\bar{\psi}=(-1)^{L_{0}} I \psi
$$

where

$$
I b_{n} I^{-1}=-\bar{b}_{n} ; \quad I \bar{b}_{n} I^{-1}=-b_{n} ; \quad I|\lambda\rangle=|-\lambda\rangle .
$$




\section{The $\mathbb{Z}_{3}$-Twisted Theory}

As in the $\mathbf{Z}_{2}$ case described in [15], the idea [24] is that we take the projection of the untwisted theory onto the $\theta=1$ sub-theory, $\mathcal{H}_{0}$, add in two twisted sectors (for a third order twist), $\mathcal{H}_{1}$ and $\mathcal{H}_{2}$, which form modules for $\mathcal{H}_{0}$, and then define appropriate vertex operators mapping between the sectors to make the whole structure a consistent conformal field theory, as defined in section 2 .

\subsection{Hilbert Space Structure}

We introduce two sets of twisted oscillators $c_{r}^{i}, \bar{c}_{r}^{i}, 1 \leq i \leq d, r \in \mathbf{Z} \pm \frac{1}{3}$. These satisfy

$$
\begin{aligned}
{\left[c_{r}^{i}, c_{s}^{j}\right] } & =\left[\bar{c}_{r}^{i}, \bar{c}_{s}^{j}\right]=r \delta^{i j} \delta_{r,-s} \\
{\left[c_{r}^{i}, \bar{c}_{s}^{j}\right] } & =0 \\
{\left[b_{m}^{i}, c_{r}^{j}\right] } & =\left[b_{m}^{i}, \bar{c}_{r}^{j}\right]=0 \\
{\left[\bar{b}_{m}^{i}, c_{r}^{j}\right] } & =\left[\bar{b}_{m}^{i}, \bar{c}_{r}^{j}\right]=0
\end{aligned}
$$

with $c_{r}^{i \dagger}=c_{-r}^{i}, \bar{c}_{r}^{i \dagger}=\bar{c}_{-r}^{i}$.

The two twisted sectors $\mathcal{H}_{1}$ and $\mathcal{H}_{2}$ are built up by the action of the oscillators $c_{r}^{i}, \bar{c}_{r}^{i}$ respectively on a representation space for a set of twisted cocycle operators (see below) annihilated by $c_{r}^{i}$ (respectively $\bar{c}_{r}^{i}$ ) for $r>0$.

\subsection{Vertex Operators Corresponding to States in the Untwisted Sector}

Let us concentrate on $\mathcal{H}_{1}$. We will define vertex operators $V_{1}(\psi, z): \mathcal{H}_{1} \rightarrow \mathcal{H}_{1}$ corresponding to states $\psi$ in the untwisted sector $\mathcal{H}_{0}$. As in the $\mathbf{Z}_{2}$ case [15], we will define $V_{1}$ initially by analogy (as in [24]) with the vertex operators (12) in $\mathcal{H}_{0}$, which we now relabel $V_{0}$, but will then find that we are forced to correct the operators in order to circumvent a normal ordering problem.

The operators

$$
L_{n}=\sum_{r \in \mathbf{Z}+\frac{1}{3}}: c_{r} \cdot c_{n-r}:+\frac{d}{9} \delta_{n 0}
$$

(with the obvious normal ordering conventions) satisfy the Virasoro algebra with $c=2 d$. We assume, and must check later, that these are the moments of the vertex operator $V_{1}\left(\psi_{L}, z\right)$.

The conformal weight of the state

$$
\chi=\prod_{a=1}^{M} c_{-r_{a}}^{j_{a}} \chi_{0}
$$


where $\chi_{0}$ is an element of the twisted sector ground state, is then

$$
h_{\chi}=\frac{d}{9}+\sum_{a=1}^{M} r_{a}
$$

So, for integral conformal weights/a meromorphic representation, we require both that $d \in 3 \mathbf{Z}$ and also that we project the twisted sector on to states with $\theta=1$, where

$$
\begin{gathered}
\theta c_{r}^{i} \theta^{-1}=e^{2 \pi i r} c_{r}^{i} \\
\theta \chi_{0}=e^{-2 p i i d / 9} \chi_{0}
\end{gathered}
$$

will give an extension of the third order automorphism from $\mathcal{H}_{0}$ to $\mathcal{H}_{1}$.

We define, for the state $\psi$ given by (11),

$$
\tilde{V}_{1}(\psi, z)=: \prod_{a=1}^{M} \frac{i}{\left(m_{a}-1\right) !} \frac{d^{m_{a}}}{d z^{m_{a}}} C_{+}^{i_{a}}(z) \prod_{b=1}^{N} \frac{i}{\left(n_{b}-1\right) !} \frac{d^{n_{b}}}{d z^{n_{b}}} C_{-}^{j_{b}}(z) e^{i \frac{\bar{\lambda}}{\alpha} \cdot C_{+}(z)} e^{i \frac{\lambda}{\alpha} \cdot C_{-}(z)}: \gamma_{\lambda}
$$

by analogy with (12), where

$$
C_{ \pm}(z)=i \sum_{r \in \mathbf{Z} \pm \frac{1}{3}} \frac{c_{r}}{r} z^{-r}
$$

and the $\gamma_{\lambda}$ are cocycle operators. (We use the obvious normal ordering convention.) Note that $\theta$ is defined so that

$$
\theta \widetilde{V}_{1}(\psi, z) \theta^{-1}=\tilde{V}_{1}(\theta \psi, z)
$$

provided that it has the appropriate action on the twisted cocycles, i.e. $\gamma_{\lambda}=\gamma_{\omega \lambda}$ for all $\lambda \in \Lambda$.

[Similarly, for $\mathcal{H}_{2}$ we define

$$
\widetilde{V}_{2}(\psi, z)=: \prod_{a=1}^{M} \frac{i}{\left(m_{a}-1\right) !} \frac{d^{m_{a}}}{d z^{m_{a}}} \bar{C}_{-}^{i_{a}}(z) \prod_{b=1}^{N} \frac{i}{\left(n_{b}-1\right) !} \frac{d^{n_{b}}}{d z^{n_{b}}} \bar{C}_{+}^{j_{b}}(z) e^{i \frac{\bar{\lambda}}{\alpha} \cdot \bar{C}_{-}(z)} e^{i \frac{\lambda}{\alpha} \cdot \bar{C}_{+}(z)}: \gamma_{\lambda}
$$

where $\bar{C}_{ \pm}$is defined following (28). If we set

$$
\theta \bar{c}_{r}^{i} \theta^{-1}=e^{-2 \pi i r} \bar{c}_{r}^{i}
$$

and

$$
\theta \chi_{0}^{\prime}=e^{2 \pi i d / 9} \chi_{0}^{\prime}
$$

for $\chi_{0}^{\prime}$ in the ground state of $\mathcal{H}_{2}$, then we see that the analogue of (29) holds. (See 28] (page 132) for a discussion of the necessity of introducing a second twisted sector.]

We now rewrite $\widetilde{V}_{1}$ in the form of a matrix element, allowing us both to see clearly a problem with its commutation relation with $L_{-1}$ and, at the same time, to correct it. We try to write

$$
\tilde{V}_{1}(\psi, z)=\sum_{\mu \in \Lambda}\left\langle\mu\left|: e^{B_{1}(-z)}:\right| \psi\right\rangle \gamma_{\mu}
$$


(which is linear in $\psi$, as required), where

$$
: e^{B_{1}(z)}:=e^{B_{1}^{-}(z)} e^{B_{1}^{+}(z)}
$$

and

$$
\begin{aligned}
& B_{1}^{+}(z)=\sum_{\substack{n \geq 0 \\
r>0}} B_{n r}^{+}(z) c_{r} \cdot \bar{b}_{n}+\sum_{\substack{n \geq 0 \\
s>0}} B_{n s}^{+}(z) c_{s} \cdot b_{n} \\
& B_{1}^{-}(z)=\sum_{\substack{n \geq 0 \\
r>0}} B_{n r}^{-}(z) c_{-r} \cdot b_{n}+\sum_{\substack{n \geq 0 \\
s>0}} B_{n s}^{-}(z) c_{-s} \cdot \bar{b}_{n} .
\end{aligned}
$$

[From now on, we shall use $r$ to indicate an element of $\mathbf{Z}+\frac{1}{3}, s$ to indicate an element of $\mathbf{Z}-\frac{1}{3}$ and $t$ for an element of $\mathbf{Z} \pm \frac{1}{3}$.] (33) is explicitly normal ordered, and a trivial calculation, analogous to that given in [15], gives

$$
B_{n t}^{ \pm}(z)=\mp \frac{1}{t}\left(\begin{array}{c}
\mp t \\
n
\end{array}\right)(-z)^{-n \mp t}
$$

[The expression for $\widetilde{V}_{2}(\psi, z)$ is obtained by making the substitution $c \rightarrow \bar{c}$ and interchanging $b$ and $\bar{b}$.]

Now, it is a simple consequence [15] of the axioms laid down in section 2 that the vertex operators $V(\psi, z)$ of a conformal field theory satisfy

$$
\left[L_{-1}, V(\psi, z)\right]=\frac{d}{d z} V(\psi, z)
$$

Following the calculation of 15 for the $\mathbf{Z}_{2}$ case, we find that this is not true of the $\tilde{V}_{1}(\psi, z)$ (with the proposed expression for $L_{-1}$ in the twisted sector). The problem is that

$$
\left[L_{-1}, c_{r}^{i}\right]=-r c_{r-1}^{i}
$$

and so terms involving $c_{\frac{1}{3}}^{i}$ and $c_{\frac{2}{3}}^{i}$ then present problems, in that they produce additional unwanted terms when normal ordered after commutation on passing through $c_{\frac{2}{3}}^{i}$ and $c_{\frac{1}{3}}^{i}$ respectively. This is corrected in a completely analogous way to that in the $\mathbf{Z}_{2}$ case, by defining

$$
V_{1}(\psi, z)=\tilde{V}_{1}\left(e^{A_{1}(-z)} \psi, z\right)
$$

where

$$
\begin{aligned}
A_{1}(z) & =\sum_{n, m \geq 0} A_{n m}^{1}(z) b_{n} \cdot \bar{b}_{m} \\
& =\sum_{\substack{n, m \geq 0 \\
n+m>0}} \frac{1}{n+m}\left(\begin{array}{c}
-\frac{2}{3} \\
n
\end{array}\right)\left(\begin{array}{c}
-\frac{1}{3} \\
m
\end{array}\right) b_{n} \cdot \bar{b}_{m}(-z)^{-n-m}-\ln (-A z) p \cdot \bar{p},
\end{aligned}
$$


and $A$ is an arbitrary constant (of integration), to be later fixed by consideration of the representation property for the twisted sectors, i.e. the relevant intertwining relations. (Note that, though this may be equivalent to the uncorrected form for $V_{1}$ with a suitable redefinition of normal ordering, it has the advantage of giving an explicit form for the vertex operators - a property which proves to be invaluable in the suvbsequent calculations.

The expression for $V_{2}(\psi, z)$ is again found by interchanging $b$ and $\bar{b}$. (Note that we use the same constant $A$ in both cases, as indeed we use the same set of twisted cocycles. That this is consistent will become clear.)

A full understanding of why this procedure works, i.e. why merely correcting the $L_{-1}$ commutation relation is sufficient to give the correct form for the vertex operators, remains to be found.

Note that $V_{1}\left(\psi_{L}, z\right)=\widetilde{V}_{1}\left(\psi_{L}, z\right)+\frac{d}{9} z^{-2}$, with modes as given in (23), justifying our earlier assumption.

The twisted conformal field theory which we are constructing is built upon the Hilbert space $\widehat{\mathcal{H}} \equiv \mathcal{H}_{0} \oplus \mathcal{H}_{1} \oplus \mathcal{H}_{2}$ (on which $\theta=1$ ). We postulate that the vertex operator corresponding to the state $(\psi, 0,0)$ (often written as $\psi$ for notational convenience) is

$$
\mathcal{V}(\psi, z)=\left(\begin{array}{ccc}
V_{0}(\psi, z) & 0 & 0 \\
0 & V_{1}(\psi, z) & 0 \\
0 & 0 & V_{2}(\psi, z)
\end{array}\right)
$$

It is easily demonstrated (c.f. [15) that we have a hermitian structure on these operators (provided the twisted cocycles behave appropriately under hermitian conjugation), i.e.

$$
\mathcal{V}(\bar{\psi}, z)=\mathcal{V}\left(e^{z^{*} L_{1}} z^{*-2 L_{0}} \psi, 1 / z^{*}\right)^{\dagger}
$$

where $\bar{\psi}$ is given by $(20)$.

\subsection{Remaining Vertex Operators}

In order to complete the verification of $\widehat{\mathcal{H}}$ as a conformal field theory, we must define vertex operators corresponding to a general state, i.e. including non-zero components in the twisted sectors.

We write

$$
\mathcal{V}\left(\left(\psi, \chi, \chi^{\prime}\right), z\right)=\left(\begin{array}{ccc}
V_{0}(\psi, z) & \bar{W}_{1}\left(\chi^{\prime}, z\right) & \bar{W}_{2}(\chi, z) \\
W_{1}(\chi, z) & V_{1}(\psi, z) & \bar{W}_{3}\left(\chi^{\prime}, z\right) \\
W_{2}\left(\chi^{\prime}, z\right) & W_{3}(\chi, z) & V_{2}(\psi, z)
\end{array}\right)
$$

If the resulting theory is to have a hermitian structure, we must have

$$
\bar{W}_{i}(\bar{\chi}, z)=W_{i}\left(e^{z^{*} L_{1}} z^{*-2 L_{0}} \chi, 1 / z^{*}\right)^{\dagger}
$$

for $i=1,2,3$ and some anti-linear involution $\chi \mapsto \bar{\chi}$ from $\mathcal{H}_{1} \rightarrow \mathcal{H}_{2}$. 
We will obviously take the preferred states to be $(|0\rangle, 0,0)$ and $\left(\psi_{L}, 0,0\right)$. The creation axiom then simply gives us

$$
V_{0}(\psi, z)|0\rangle=e^{z L_{-1}} \psi
$$

which holds as it is satisfied in the untwisted conformal field theory,

$$
W_{1}(\chi, z)|0\rangle=e^{z L_{-1}} \chi
$$

and

$$
W_{2}\left(\chi^{\prime}, z\right)|0\rangle=e^{z L_{-1}} \chi^{\prime}
$$

The only remaining axiom to verify, and which must furnish us with sufficient information to define $W_{1}, W_{2}$ and $W_{3}$ (and the conjugation map on the twisted states), is the locality axiom. The locality relations for the operators $\mathcal{V}(\rho, z), \rho \in \widehat{\mathcal{H}}$, are seen to be equivalent to 18 distinct locality relations for the component vertex operators. Many of these are hermitian conjugates of others. We consider the 12 that remain, i.e.

$$
\begin{aligned}
V_{0}(\psi, z) V_{0}(\phi, w) & =V_{0}(\phi, w) V_{0}(\psi, z) \\
V_{1}(\psi, z) V_{1}(\phi, w) & =V_{1}(\phi, w) V_{1}(\psi, z) \\
V_{2}(\psi, z) V_{2}(\phi, w) & =V_{2}(\phi, w) V_{2}(\psi, z) \\
W_{1}(\chi, z) V_{0}(\psi, w) & =V_{1}(\psi, w) W_{1}(\chi, z) \\
W_{2}\left(\chi^{\prime}, z\right) V_{0}(\psi, w) & =V_{2}(\psi, w) W_{2}\left(\chi^{\prime}, z\right) \\
\bar{W}_{1}\left(\chi^{\prime}, z\right) W_{1}(\chi, w) & =\bar{W}_{2}(\chi, w) W_{2}\left(\chi^{\prime}, z\right) \\
W_{3}(\chi, z) V_{1}(\psi, w) & =V_{2}(\psi, w) W_{3}(\chi, z) \\
W_{3}(\chi, z) W_{1}(\rho, w) & =W_{3}(\rho, w) W_{1}(\chi, z) \\
\bar{W}_{3}\left(\chi^{\prime}, z\right) W_{3}(\chi, w) & =W_{1}(\chi, w) \bar{W}_{1}\left(\chi^{\prime}, z\right) \\
W_{3}(\chi, z) \bar{W}_{3}\left(\chi \chi^{\prime}, w\right) & =W_{2}(\chi, w) \bar{W}_{2}(\chi, z) \\
\bar{W}_{2}(\chi, z) W_{3}(\rho, w) & =\bar{W}_{2}(\rho, w) W_{3}(\chi, z) \\
W_{1}(\chi, z) \bar{W}_{2}(\rho, w) & =W_{1}(\rho, w) \bar{W}_{2}(\chi, z)
\end{aligned}
$$

for $\psi, \phi \in \mathcal{H}_{0}, \chi, \rho \in \mathcal{H}_{1}$ and $\chi^{\prime}, \rho^{\prime} \in \mathcal{H}_{2}$. We know (48) from the fact that the $V_{0}$ 's specify a sub-theory of a consistent conformal field theory.

Consider the action of (51) and (52) on $|0\rangle$, and use (45-47). We find (taking $w=0$ )

$$
W_{1}(\chi, z) \psi=V_{1}(\psi, 0) e^{z L_{-1}} \chi
$$

or

$$
W_{1}(\chi, z) \psi=e^{z L_{-1}} V_{1}(\psi,-z) \chi
$$

from (exponentiation of) (37), and similarly

$$
W_{2}\left(\chi^{\prime}, z\right) \psi=e^{z L_{-1}} V_{2}(\psi,-z) \chi^{\prime} .
$$

Thus, $W_{1}$ and $W_{2}$ are fixed uniquely by the requirements of locality, as was the intertwiner in the $\mathbf{Z}_{2}$ case. The definition of $W_{3}$, the intertwiner of the twisted sectors, is however more intricate, and we will discuss this new feature in a later section.

In the following sections, we will consider the above locality relations in some detail. 


\subsection{Representation Property}

Firstly, we will verify (49-52).

We show that (49) follows from (51). Consider

$$
\begin{array}{rlr}
V_{1}(\psi, z) V_{1}(\phi, w) \chi & =V_{1}(\psi, z) V_{1}(\phi, w) W_{1}(\chi, 0)|0\rangle & \\
& =W_{1}(\chi, 0) V_{0}(\psi, z) V_{0}(\phi, w)|0\rangle & \text { by }(51) \\
& =W_{1}(\chi, 0) V_{0}(\phi, w) V_{0}(\psi, z)|0\rangle & \text { by (48) } \\
& =V_{1}(\phi, w) V_{1}(\psi, z) W_{1}(\chi, 0)|0\rangle & \text { by (51) } \\
& =V_{1}(\phi, w) V_{1}(\psi, z) \chi, &
\end{array}
$$

as required. Similarly, (50) is a consequence of (52). We therefore check only (51) and (52).

[Note that [14]

$$
\begin{aligned}
V_{1}(\psi, z) V_{1}(\phi, w) \chi & =W_{1}(\chi, 0) V_{0}(\psi, z) V_{0}(\phi, w)|0\rangle \quad \text { from above } \\
& =W_{1}(\chi, 0) V_{0}\left(V_{0}(\psi, z-w) \phi, w\right)|0\rangle
\end{aligned}
$$

by the duality property of the conformal field theory $\mathcal{H}_{0}$ (see e.g. [23])

$$
\begin{aligned}
& =V_{1}\left(V_{0}(\psi, z-w) \phi, w\right) W_{1}(\chi, 0)|0\rangle \quad \text { by (51) } \\
& =V_{1}\left(V_{0}(\psi, z-w) \phi, w\right) \chi
\end{aligned}
$$

i.e.

$$
V_{1}(\psi, z) V_{1}(\phi, w)=V_{1}\left(V_{0}(\psi, z-w) \phi, w\right),
$$

and we say that $\mathcal{H}_{1}$ is a representation of the conformal field theory $\mathcal{H}_{0}$ [14]. Similarly, $\mathcal{H}_{2}$ also forms a module for $\mathcal{H}_{0}$.]

Consider now the locality relation (51). We write $V_{0}(\psi, z)$ in matrix element form:-

$$
V_{0}(\psi, z)=\sum_{\mu^{\prime} \in \Lambda}\left\langle\mu^{\prime}\left|e^{i \bar{p}^{\prime} \cdot q} e^{i p^{\prime} \cdot \bar{q}}: e^{M(z)}:\right| \psi^{\prime}\right\rangle \sigma_{\mu}^{\prime},
$$

where

$$
: e^{M(z)}:=e^{M^{-}(z)} e^{M^{+}(z)},
$$

with

$$
\begin{aligned}
& M^{+}(z)=\sum_{n, m \geq 0} M_{n m}^{+}(z) b_{n}^{\prime} \cdot \bar{b}_{m}+\sum_{n, m \geq 0} M_{n m}^{+}(z) \bar{b}_{n}^{\prime} \cdot b_{m} \\
& M^{-}(z)=\sum_{\substack{n \geq 0 \\
m>0}} M_{n m}^{-}(z) b_{n}^{\prime} \cdot \bar{b}_{-m}+\sum_{\substack{n \geq 0 \\
m>0}} M_{n m}^{-}(z) \bar{b}_{n}^{\prime} \cdot b_{-m},
\end{aligned}
$$

the ' indicating a second (commuting) copy of $\mathcal{H}_{0}$. A simple argument (c.f. 15]) gives

$$
M_{n m}^{ \pm}(z)=\mp \frac{1}{m}\left(\begin{array}{c}
\mp m \\
n
\end{array}\right) z^{\mp m-n},
$$


for $n \geq 0, m>0$, and

$$
\begin{gathered}
M_{00}^{+}(z)=\ln z \\
M_{n 0}^{+}(z)=-\frac{1}{n}(-z)^{-n},
\end{gathered}
$$

for $n>0$.

Suppose that

$$
\gamma_{\lambda} \gamma_{\mu}=\epsilon(\lambda, \mu) e^{i S(\lambda, \mu)} \gamma_{\lambda+\mu}
$$

where

$$
\hat{\sigma}_{\lambda} \hat{\sigma}_{\mu}=\epsilon(\lambda, \mu) \hat{\sigma}_{\lambda+\mu}
$$

i.e. we allow for a possible difference in the symmetry factors for the straight and twisted sector cocycle operators. This gives

$$
\gamma_{\lambda} \gamma_{\mu}=\frac{\epsilon(\lambda, \mu)}{\epsilon(\mu, \lambda)} e^{P(\lambda, \mu)} \gamma_{\mu} \gamma_{\lambda}
$$

where

$$
P(\lambda, \mu) \equiv i(S(\lambda, \mu)-S(\mu, \lambda))
$$

Thus,

$$
\gamma_{\lambda} \gamma_{\mu}=(-1)^{\langle\lambda, \mu\rangle / \alpha^{2}} e^{P(\lambda, \mu)} \gamma_{\mu} \gamma_{\lambda}
$$

using (74) together with (16).

From (61), (39) and (33) we have

$$
W_{1}(\chi, z)=e^{z L_{-1}} \sum_{\mu \in \Lambda}\left\langle\mu\left|: e^{B_{1}(z)}: e^{A_{1}(z)}\right| \chi\right\rangle \gamma_{\mu} .
$$

Hence (51) becomes

$$
\sum_{\mu \in \Lambda} \gamma_{\mu}\left\langle\mu\left|: e^{B_{1}(z)}: e^{A_{1}(z)}\right| \chi\right\rangle V_{0}(\psi, z)=V_{1}(\psi, w-z) \sum_{\mu \in \Lambda} \gamma_{\mu}\left\langle\mu\left|: e^{B_{1}(z)}: e^{A_{1}(z)}\right| \chi\right\rangle
$$

using (exponentiation of) (37), or from (66), (39) and (33)

$$
\begin{aligned}
& \sum_{\mu \in \Lambda} \gamma_{\mu}\left\langle\mu\left|: e^{B_{1}(z)}: e^{A_{1}(z)}\right| \chi\right\rangle \sum_{\mu^{\prime} \in \Lambda}\left\langle\mu^{\prime}\left|e^{i \bar{p}^{\prime} \cdot q} e^{i p^{\prime} \cdot \bar{q}}: e^{M(w)}:\right| \psi^{\prime}\right\rangle \sigma_{\mu^{\prime}} \\
= & \sum_{\mu^{\prime} \in \Lambda} \gamma_{\mu^{\prime}}\left\langle\mu^{\prime}\left|: e^{B_{1}(z-w)}: e^{A_{1}(z-w)}\right| \psi^{\prime}\right\rangle \sum_{\mu \in \Lambda} \gamma_{\mu}\left\langle\mu\left|: e^{B_{1}(z)}: e^{A_{1}(z)}\right| \chi\right\rangle .
\end{aligned}
$$

We simply reorder the exponentials on the right-hand side by use of the Baker-CampbellHausdorff identities (see [15] for an analogous calculation in the $\mathbf{Z}_{2}$ case). Then (80) is found to be equivalent to the following requirements:-

$$
\left[B_{1}^{ \pm}(\zeta), \widetilde{M}^{-}(z)\right]=B_{1}^{ \pm}(-(z-\zeta))^{\prime}
$$




$$
\begin{gathered}
\frac{1}{2}\left[\widetilde{M}^{-}(-z),\left[A_{1}(\zeta), \widetilde{M}^{-}(-z)\right]\right]=A_{1}(-(z-\zeta))^{\prime} \\
\left\langle\lambda^{\prime}\right|\langle\mu|\left\{\left[B_{1}^{-}(\zeta),\left[B_{1}^{+}(\zeta), \widetilde{M}^{-}(-z)\right]\right]+\left[A_{1}(\zeta), \widetilde{M}^{-}(-z)\right]+M^{+}(-z)\right\}=\left\langle\lambda^{\prime}\right|\langle\mu| i S(\lambda, \mu),
\end{gathered}
$$

where

$$
\widetilde{M}^{-}(z)=M^{-}(z)+i \bar{p}^{\prime} \cdot q+i p^{\prime} \cdot \bar{q}
$$

and $B_{1}^{ \pm}(z)^{\prime}, A_{1}(z)^{\prime}$ denote $B_{1}^{ \pm}(z), A_{1}(z)$ respectively written in terms of the primed oscillators.

Note the occurrence of the phase factor $S(\lambda, \mu)$ in (83). In the $\mathbf{Z}_{2}$-twisted case, we were able to choose the arbitrary constant of integration in our definition of $A(z)$ such that this term was absent. However, in this case we see that in general we shall not be able to choose this constant to cancel both the terms in $p^{\prime} \cdot \bar{p}$ and those in $\bar{p}^{\prime} \cdot p$ inside the $\{\ldots\}$ of (83). What we shall do is to choose $A$ so that $S(\lambda, \mu)$ is real, which is consistent with a representation of the twisted cocycles by unitary matrices.

The identities 81-83) may be checked by replacing $b_{n}$ by $x^{n}, b_{n}^{\prime}$ by $y^{n}, \bar{b}_{n}$ by $\bar{x}^{n}$ and $\bar{b}_{n}^{\prime}$ by $\bar{y}^{n}$ and then summing to obtain the generating functions for the coefficients of the terms involving these oscillators. [Note that, in general, this procedure will be unable to distinguish between say $b_{-1}^{n}$ and $b_{-n}$, though because we know the form of the operators it is sufficient in this particular case.] The simple identities which are required are

$$
\begin{gathered}
\sum_{n, m \geq 0} x^{m} y^{n} A_{n m}^{1}(z)=-(1-\omega) \ln \left(\frac{(y-z)^{\frac{1}{3}}-\omega(x-z)^{\frac{1}{3}}}{(1-\omega)}\right) \\
-(1-\bar{\omega}) \ln \left(\frac{(y-z)^{\frac{1}{3}}-\bar{\omega}(x-z)^{\frac{1}{3}}}{(1-\bar{\omega})}\right)-\ln A \\
\sum_{n \geq 0} y^{n} B_{n t}^{ \pm}(z)=\mp \frac{1}{t}(y-z)^{\mp t} \\
\sum_{r>0} \frac{y^{r}}{r}=-\ln \left(1-y^{\frac{1}{3}}\right)-\bar{\omega} \ln \left(1-\omega y^{\frac{1}{3}}\right)-\omega \ln \left(1-\bar{\omega} y^{\frac{1}{3}}\right) \\
\sum_{s>0} \frac{y^{s}}{s}=-\ln \left(1-y^{\frac{1}{3}}\right)-\omega \ln \left(1-\omega y^{\frac{1}{3}}\right)-\bar{\omega} \ln \left(1-\bar{\omega} y^{\frac{1}{3}}\right) .
\end{gathered}
$$

(81) and (82) are easily checked, while (83) gives

$$
\begin{aligned}
i S(\lambda, \mu)=\frac{\lambda \cdot \bar{\mu}}{\alpha^{2}}\{-\ln A+(1-\bar{\omega}) \ln (1-\omega)+(1-\omega) \ln (1-\bar{\omega})\} \\
\quad+\frac{\bar{\lambda} \cdot \mu}{\alpha^{2}}\{-\ln A+(1-\omega) \ln (1-\omega)+(1-\bar{\omega}) \ln (1-\bar{\omega})\} .
\end{aligned}
$$


Hence

$$
\begin{aligned}
P(\lambda, \mu) & =\left\{\frac{(\lambda, \mu)-(\mu, \lambda)}{\alpha^{2}}\right\}(\omega-\bar{\omega}) \ln \left(\frac{1-\omega}{1-\bar{\omega}}\right) \\
& =\frac{\pi}{\sqrt{3}}\left\{\frac{(\lambda, \mu)-(\mu, \lambda)}{\alpha^{2}}\right\} .
\end{aligned}
$$

(Note that the expression for $P(\lambda, \mu)$ is independent of $A$.)

Choosing $A$ so that $S(\lambda, \mu)$ is real gives

$$
A=3 \sqrt{3} .
$$

The phase $P(\lambda, \mu)$ of (90) has already been found in [25, 26] (in the case $\alpha^{2}=3$ ), but only in the case of the locality of the twisted vertex operators corresponding to pure momentum states. The above calculation verifies the intertwining relation (and hence the representation property, by the above arguments) for arbitrary (untwisted) states. Note that the work of [25, 26] is for twists of arbitrary order, and below we shall consider the generalisation of our approach to twists of an arbitrary prime order.

\subsection{Self-duality and the definition of $\alpha$}

We now digress for a while from consideration of the locality relations to discuss the self-duality of the resulting conformal field theory.

Let us consider what condition it is necessary to impose on the Eisenstein lattice in order to produce a self-dual $\mathbf{Z}_{3}$-twisted conformal field theory, by which we mean that the partition function of the theory,

$$
\chi_{\mathcal{H}}(\tau)=\operatorname{Tr}_{\mathcal{H}} q^{L_{0}-c / 24}
$$

is invariant under the modular transformation $S: \tau \mapsto-1 / \tau$ (and hence up to a phase under a general modular transformation). (See [23] for a fuller discussion of the problem of satisfactorily defining self-duality.)

There are two clear choices, to either make $\Lambda_{R} / \alpha$ self-dual (so that the untwisted theory before projection is self-dual [15]) or to make $\Lambda$ (the "natural" structure underlying this construction) self-dual. The first of these is clearly the correct one, as it is in the orbifold spirit of starting with a self-dual theory, projecting out by an automorphism and then adding in twisted representations of the remaining theory to restore self-duality (although this does rely on naive physical ideas of how the various twisted sector contributions to the partition function and the characters of the automorphism transform into one another under modular transformations - see e.g. [37, 22]). [Note that the two choices are in general inequivalent, since

$$
\operatorname{det}\left(\Lambda_{R}\right)=3^{d} \operatorname{det} \Lambda
$$

and so they are only simultaneously self-dual for $\alpha=3^{\frac{1}{4}}$.] 
Let us for the sake of completeness show that self-duality of $\Lambda$ is inconsistent with self-duality of the corresponding twisted conformal field theory. Concentrate on the case $d=12$ (i.e. $c=24$ ), and let us evaluate the first few terms in the expansion of the partition function for the theory obtained by projecting out on to the $\theta=1$ space $\mathcal{H}_{0} \oplus \mathcal{H}_{1} \oplus \mathcal{H}_{2}$. We would expect to obtain a partition function of the form $j(\tau)+$ constant (since $j$ is the unique modular invariant function up to an additive constant with a single simple pole at the origin in $q=e^{2 \pi i \tau}$ ). Taking $\Lambda$ to be self-dual fixes its theta function [8]

$$
\Theta_{\Lambda}(\tau) \in \mathbf{C}\left[\phi_{0}(\tau / 2), \Delta_{6}(\tau)\right]
$$

where

$$
\begin{gathered}
\phi_{0}(\tau / 2)=1+6 q+6 q^{3}+6 q^{4}+12 q^{7}+\ldots \\
\Delta_{6}(\tau)=q \prod_{n=1}^{\infty}\left(1-q^{n}\right)^{6}\left(1-q^{3 n}\right)^{6}
\end{gathered}
$$

i.e.

$$
\Theta_{\Lambda}(\tau)=\phi_{0}(\tau / 2)^{12}+C_{1} \phi_{0}(\tau / 2)^{6} \Delta_{6}(\tau)+C_{2} \Delta_{6}(\tau)^{2}
$$

where $C_{1}, C_{2} \in \mathbf{C}$. Equating the partition function of the theory to $j(\tau)$ up to an additive constant and considering the first few terms in the series expansion determines the degeneracy of the twisted sector ground states (we assume the same value for the degeneracy in both sectors), and we find this to be non-integral, providing the required contradiction.

A similar calculation using $\Lambda_{R} / \alpha$ self-dual instead appears to produce a self-dual partition function (the first few coefficients are correct) provided the twisted sector ground state degeneracy takes the value $729\left(=3^{6}\right.$, which is highly plausible!).

Let us therefore investigate the twisted sector degeneracy by studying the representations of the twisted sector cocycles. Since $\Lambda$ is integral, $(\lambda, \mu) \in \mathcal{E}$. Suppose $(\lambda, \mu)=$ $m+n \omega$. Then $P(\lambda, \mu)=\pi i n / \alpha^{2}$, and $\langle\lambda, \mu\rangle=2 m-n$. Hence

$$
\gamma_{\lambda} \gamma_{\mu}=e^{2 \pi i m / \alpha^{2}} \gamma_{\mu} \gamma_{\lambda}
$$

using $(77)$.

But we have set $\operatorname{det}\left(\Lambda_{R} / \alpha\right)=1$. Hence, by (93), $\operatorname{det} \Lambda=\left(\alpha^{4} / 3\right)^{d}$. Since $\Lambda$ is integral, $\operatorname{det} \Lambda \geq 1$, and so we must have $\alpha^{2} \geq \sqrt{3}$. Consider the set of integers $m_{\lambda}=(\lambda, \lambda) / \alpha^{2}$, $\lambda \in \Lambda$. Suppose that their greatest common divisor is 1 . (This is certainly true for $\Lambda_{R} / \alpha$ a Niemeier lattice, since $m_{\lambda}$ actually takes the value 1 for all but the Leech lattice, and the result clearly holds in that case by inspection.) We know that $(\lambda, \lambda)=M_{\lambda} \in \mathbf{Z}$, since $\Lambda$ is integral. This gives us $\alpha^{2}=M_{\lambda} / m_{\lambda} \in \mathbf{Z}$. Also, we have, from the argument following equation (29), $\gamma_{\lambda}=\gamma_{\omega \lambda}$, so that identifying the phases in (98) for $\lambda$ replaced by $\omega \lambda$ we obtain the restriction $3 m \in \alpha^{2} \mathbf{Z}$. If the lattice $\Lambda$ is extremal, in the sense that $\Lambda_{\beta}=\Lambda / \beta$ does not satisfy $(\lambda, \lambda) \in n \mathbf{Z}$ for all $\lambda \in \Lambda_{\beta}$ and some (fixed) integer $n$ and factor $\beta>1$, then this is clearly equivalent to the statement that our values of $m$ above have no common factor, and so the constraint arising from the invariance of $\gamma_{\lambda}$ under the action of the lattice automorphism gives us $\alpha^{2}=3$. We shall use this value from now on. 


\subsection{Generalisation to Higher Order Symmetries}

Before continuing with our consideration of locality for the $\mathbf{Z}_{3}$-twisted theory, let us note that the generalisation to higher order symmetries $\mathbf{Z}_{p}$ is essentially obvious. (We shall take $p>3$ to be prime for simplicity, although there is no a priori reason why we must do so.) No new features arise beyond those appearing in the transition form the $\mathbf{Z}_{2}$ to the $\mathbf{Z}_{3}$ case.

We introduce $p-1$ sets of oscillators $b_{n}^{k i}, 1 \leq k \leq p-1,1 \leq i \leq d, n \in \mathbf{Z}$, and $p-1$ sets of twisted oscillators $c_{r}^{k i}, 1 \leq k \leq p-1,1 \leq i \leq d, r \in \mathbf{Z} / p-\mathbf{Z}$. These satisfy the relations

$$
\begin{gathered}
{\left[b_{m}^{k i}, b_{n}^{l j}\right]=m \delta^{i j} \delta_{m,-n} \delta_{k+l, p}} \\
{\left[c_{r}^{k i}, c_{s}^{l j}\right]=r \delta^{i j} \delta_{r,-s} \delta_{k l}}
\end{gathered}
$$

and all other commutation relations vanish, with $b_{n}^{k i \dagger}=b_{-n}^{p-k, i}, c_{r}^{k i \dagger}=c_{-r}^{k i}$. The straight sector, $\mathcal{H}_{0}$, is created by the action of the $b$-oscillators on a set of orthonormal states $|\lambda\rangle$ as usual, where $p^{k i} \equiv b_{0}^{k i}$ satisfy $p^{k i}|\lambda\rangle=\lambda_{k}|\lambda\rangle$ with $\lambda_{k}$ a $d$-dimensional complex vector and $\overline{\lambda_{k}}=\lambda_{p-k}$ (any scaling, such as that introduced by the factor $\alpha$ in the $\mathbf{Z}_{3}$ case, has been absorbed into the definition of $\lambda_{k}$ ), while we have $p-1$ twisted sectors, $\mathcal{H}_{1}, \ldots, \mathcal{H}_{p-1}$, where $\mathcal{H}_{k}$ is created by the action of the oscillators $c_{-r}^{k i}, r>0$, on some appropriate twisted cocycle representation space. The twisted sectors are to form meromorphic representations of the conformal field theory described by the subspace of the twisted sector left invariant under the action of an automorphism $\theta$ of order $p$.

The vertex operators in $\mathcal{H}_{0}$ are given by

$$
V_{0}(\psi, z)=: \prod_{k=1}^{p-1} \prod_{a=1}^{M_{k}} \frac{i}{\left(n_{k a}-1\right) !} \frac{d^{n_{k a}}}{d z^{n_{k a}}} X^{k i_{k a}}(z) \cdot \exp \left\{\sum_{k=1}^{p-1} i \lambda_{p-k} \cdot X^{k}(z)\right\}: \sigma_{\lambda}
$$

for

$$
\psi=\prod_{k=1}^{p-1} \prod_{a=1}^{M_{k}} b_{-n_{k a}}^{i_{k a}}|\lambda\rangle
$$

$\sigma_{\lambda}$ is a cocycle operator, and

$$
X^{k i}(z)=q^{k i}-i p^{k i} \ln z+i \sum_{n \neq 0} \frac{b_{n}^{k i}}{n} z^{-n}
$$

with

$$
\exp \left\{\sum_{k=1}^{p-1} \lambda_{k} \cdot q^{k}\right\}|\mu\rangle=|\lambda+\mu\rangle
$$

(addition inside the ket corresponding to addition of the corresponding eigenvalues of the operators $p^{k i}$ ), and the obvious normal ordering. 
Set $\hat{\sigma}_{\lambda}=\exp \left\{\sum_{k=1}^{p-1} \lambda_{k} \cdot q^{k}\right\} \sigma_{\lambda}$. Then these operators must obey

$$
\hat{\sigma}_{\lambda} \hat{\sigma}_{\mu}=(-1)^{\langle\lambda, \mu\rangle} \hat{\sigma}_{\mu} \hat{\sigma}_{\lambda}
$$

where the real symmetric bilinear form $\langle\cdot, \cdot\rangle$ is given by

$$
\langle\lambda, \mu\rangle \equiv \sum_{k=1}^{p-1} \lambda_{k} \cdot \mu_{p-k},
$$

in order that the $V_{0}$ be local. This demonstrates the general feature of the calculations for higher values of $p$. The operators in the untwisted sector pair up (i.e. the $k$ 'th set with the $(p-k)$ 'th set) and we essentially have $(p-1) / 2$ separate commuting calculations to perform, leading to the $(p-1) / 2$ separate terms in (106) of the form $\lambda_{k} \cdot \mu_{p-k}+\lambda_{p-k} \cdot \mu_{k}$. The case $p=2$ is distinct, as we have one set of oscillators only, which pairs with itself. This is the origin of the new feature noted above in the symmetry factors for the twisted operator cocycles.

The Virasoro state

$$
\psi_{L}=\frac{1}{2} \sum_{k=1}^{p-1} b_{-1}^{k} b_{-1}^{p-k}|0\rangle,
$$

and the moments of the corresponding vertex operator

$$
L_{n}=\frac{1}{2}: \sum_{k=1}^{p-1} \sum_{m=-\infty}^{\infty} b_{m}^{k} \cdot b_{n-m}^{p-k}:
$$

satisfy the Virasoro algebra with $c=(p-1) d$. The conformal weight of the state (102) is given by

$$
\begin{aligned}
h_{\psi} & =\frac{1}{2} \sum_{k=1}^{p-1} \lambda_{k} \cdot \lambda_{p-k}+\sum_{k=1}^{p-1} \sum_{a=1}^{M_{k}} n_{k a} \\
& =\frac{1}{2}\langle\lambda, \lambda\rangle+\sum_{k=1}^{p-1} \sum_{a=1}^{M_{k}} n_{k a} .
\end{aligned}
$$

Hence, the theory will be meromorphic provided $\langle\cdot, \cdot\rangle$ defines an even $(p-1) d$-dimensional Z-lattice. (Clearly, we expect the theory on $\mathcal{H}_{0}$ before projection to be self-dual if this lattice is self-dual, i.e. $(p-1) d$ must be a multiple of 8 .)

Define

$$
\theta b_{n}^{k i} \theta^{-1}=\omega_{p}^{k} b_{n}^{k i} .
$$

From (101) we see that, for $\theta$ to be an automorphism of the theory on $\mathcal{H}_{0}$, we must have

$$
\theta \lambda_{k}=\omega_{p}^{-k} \lambda_{k} .
$$


The operators

$$
L_{n k}=\frac{1}{2}: \sum_{l=1}^{p-1} \sum_{r \in \mathbf{Z}+\frac{l}{p}} c_{r}^{k} \cdot c_{n-r}^{k}:+\delta_{n 0} \frac{\left(p^{2}-1\right) d}{24 p}
$$

satisfy the Virasoro algebra with $c=d(p-1)$. We assume for the moment that these are the modes of the relevant twisted sector vertex operator. Hence, the conformal weight of the twisted sector ground state is $\frac{\left(p^{2}-1\right) d}{24 p}$. This must lie in $\mathbf{Z} / p$ in order that we may ultimately obtain a meromorphic representation. Hence, we require $24 \mid\left(p^{2}-1\right) d$. As remarked above, for $\mathcal{H}_{0}$ to be a self-dual theory, $(p-1) d \in 8 \mathbf{Z}$. Say $(p-1) d=8 N$. Then we require $3 \mid(p+1) N$. So $c=(p-1) d$ must be a multiple of 24 if $3 \not(p+1)$, otherwise it must only be a multiple of 8 .

Set

$$
\begin{gathered}
\theta c_{-r}^{k i} \theta^{-1}=\omega_{p}^{\sigma_{r}} c_{-r}^{k i} \\
\theta \chi_{k}=\omega_{p}^{\rho_{k}} \chi_{k}
\end{gathered}
$$

where $\chi_{k}$ is an element of the ground state of the twisted sector $\mathcal{H}_{k}, 1 \leq k \leq p-1$, and $\sigma_{r}, \rho_{k} \in \mathbf{Z}$. In order that the projection on to $\theta=1$ states provides a meromorphic theory, we require

$$
\sigma_{r_{1}}+\ldots+\sigma_{r_{M}}+\rho_{k} \in p \mathbf{Z} \Longleftrightarrow r_{1}+\ldots+r_{M}+\frac{\left(p^{2}-1\right) d}{24 p} \in \mathbf{Z}
$$

i.e. we take

$$
\begin{gathered}
\sigma_{r}=k p r \\
\rho_{k}=\frac{k N(p+1)}{3} .
\end{gathered}
$$

The vertex operators $V_{k}(\psi, z)$ in the sector $\mathcal{H}_{k}$ corresponding to a state $\psi \in \mathcal{H}_{0}$ are defined as before, i.e. by writing down a naive form and then correcting it by considering the $L_{-1}$ commutation relation. Note that this introduces $\frac{p-1}{2}$ arbitrary constants, and these can be chosen so that the additional contributions to $\epsilon(\lambda, \mu)$ in the twisted sector are pure phases, as we did above, by choosing the coefficients of the $p^{k^{\prime}} \cdot p^{p-k}$ and $p^{p-k^{\prime}} \cdot p^{k}$ terms to be equal and opposite. Set

$$
C_{l}^{k}(z)=i \sum_{r \in \mathbf{Z}+\frac{l}{p}} \frac{c_{r}^{k}}{r} z^{-r}
$$

The couplings between the $b$-oscillators and the $C_{l}^{k}(z)$ in $V_{k}(\psi, z)$ are given by requiring $\theta$ to be an automorphism, i.e. suppose $b^{l} \leftrightarrow C_{\mu_{k}(l)}^{k}(z)$. Then $\theta$ is an automorphism, from (110) and (113), if $l=\sigma_{-\mu_{k}(l) / p}$, i.e. $l=-k \mu_{k}(l)$ from (116). Thus $\mu_{k}(l)=-l k^{p-2}$ (using $k^{p-1} \equiv 1 \bmod p$ for $\left.k \not \equiv 0 \bmod p\right)$. We may proceed as before, and check the representation property of the twisted sectors by verifying the intertwining locality relation. Note that 
we need only check the relation for one twisted sector, and also that we need merely to check the relevant analogues of (81-83). This decomposes into $\frac{p-1}{2}$ copies of these relations, which then reduce to (85-88) with $\frac{1}{3}, \frac{2}{3}$ replaced by $\frac{k}{p}, \frac{p-k}{p}$ for $k=1, \ldots, \frac{p-1}{2}$. We recover the cocycle phase found in [25, 26], but, as before, we have extended the proof of locality to the full Hilbert space, rather then just the momentum states.

In the investigation below of the remaining locality relations, we shall consider the case $p=3$ for simplicity. But, as we have seen in the above, the generalisation to higher values of $p$ is trivial (only the case $p=2$ is special).

\subsection{Further locality checks}

\subsubsection{Twisted Sector Conjugation}

The next locality relation in turn, i.e. (53), is analogous to the relation

$$
\bar{W}(\chi, z) W(\rho, w)=\bar{W}(\rho, w) W(\chi, z)
$$

in the $\mathbf{Z}_{2}$ case [15], in which it was found to be equivalent to a reality condition on the representation - a relation which the conjugation map on the twisted sector had to be chosen to satisfy. An identical argument holds here, and we find that the locality relation is equivalent to

$$
\left(f_{\chi_{1} \phi \chi_{2}}\right)^{*}=(-1)^{h_{1}+h_{\phi}+h_{2}} f_{\bar{\chi}_{1} \bar{\phi} \bar{\chi}_{2}}
$$

where

$$
f_{\chi_{1} \phi \chi_{2}}=\left\langle\bar{\chi}_{1}\left|V_{i}(\phi, 1)\right| \chi_{2}\right\rangle
$$

with either $i=1, \chi_{1} \in \mathcal{H}_{1}, \chi_{2} \in \mathcal{H}_{2}$ or $i=2, \chi_{1} \in \mathcal{H}_{2}, \chi_{2} \in \mathcal{H}_{1}$, and $L_{0} \phi=h_{\phi} \phi$, $L_{0} \chi_{j}=h_{j} \chi_{j}$ for $j=1,2$. We see that if we take

$$
\bar{\chi}=(-1)^{L_{0}} I \chi
$$

for $\chi \in \mathcal{H}_{1} \cup \mathcal{H}_{2}$ with

$$
I c_{r} I^{-1}=-\bar{c}_{r} ; \quad I \bar{c}_{r} I^{-1}=-c_{r} ; \quad I \chi_{0}=M \chi_{0}^{*}
$$

for $\chi_{0}$ a twisted sector ground state, where

$$
M^{\dagger} \gamma_{\lambda} M=\gamma_{\lambda}^{*}
$$

with $M$ a symmetric unitary matrix, then $(120)$ is satisfied.

\subsubsection{Definition of $W_{3}(\chi, z)$}

In the case of intertwiners between twisted sectors, one cannot use our previous trick of using action on the vacuum and the creation axiom. We must instead take a more indirect approach. 
Assuming that $W_{3}(\chi, z)$ satisfies the intertwining relation (54) and we have the duality relation for the operators $\mathcal{V}(\rho, z)$ (which must hold if they are to define a consistent conformal field theory), i.e.

$$
\mathcal{V}(\rho, z) \mathcal{V}(\phi, w)=\mathcal{V}(\mathcal{V}(\rho, z-w) \phi, w)
$$

(c.f. (65)), then

$$
\begin{aligned}
W_{3}\left(V_{1}\left(\phi_{1}, w_{1}\right) \chi_{0}, z\right) V_{1}\left(\phi_{2}, w_{2}\right) \chi_{0} & =V_{2}\left(\phi_{2}, w_{2}\right) W_{3}\left(V_{1}\left(\phi_{1}, w_{1}\right) \chi_{0}, z\right) \chi_{0} \\
& =V_{2}\left(\phi_{2}, w_{2}\right) V_{2}\left(\phi_{1}, w_{1}+z\right) W_{3}\left(\chi_{0}, z\right) \chi_{0},
\end{aligned}
$$

where $\chi_{0} \in \mathcal{H}_{1}$ and $\phi_{1}, \phi_{2} \in \mathcal{H}_{0}$. Since $\mathcal{H}_{1}$ is irreducible as a representation space for the theory described by the untwisted sector, then we may take (126) as the definition of $W_{3}(\chi, z)$ in terms of an unknown state $F_{\chi_{0}}(z) \equiv W_{3}\left(\chi_{0}, z\right) \chi_{0} \in \mathcal{H}_{2}$ with $\chi_{0}$ a (fixed) element of the twisted sector ground state.

Note that $\chi_{0}$ lies in the projected theory only when $c$ is a multiple of 72 , but we expect this to make little difference to the arguments, i.e. we would expect a verification of the remaining locality relations for $c$ a multiple of 72 to extend to other values of $c$ without any problem.

The definition (126) does not give an explicit form for $W_{3}(\chi, z)$. Indeed, constructing an explicit form once $F_{\chi_{0}}(z)$ is known is non-trivial, since it involves inverting the action of $V_{1}(\psi, z)$ on $\mathcal{H}_{1}$. One of the advantages of our approach in [15, 16, 14 over that of FLM is that it gives explicit forms for many operators whose properties FLM were forced to infer by other considerations. This is here to some extent lost. In addition, we would expect, from the symmetry of the nature of the construction, that $\bar{W}_{3}(\chi, z)$ would be of a similar form to $W_{3}(\chi, z)$, simply with the $c$ - and $\bar{c}$-oscillators interchanged. This symmetry would be apparent if an explicit form for $W_{3}(\chi, z)$ were given, but is obscured by our choice of definition (although in Appendix A we do provide a proof in the presence of the full locality relations of the theory, i.e. assuming the conformal field theory to be consistent). Still, in the absence of a natural definition (c.f. the definition of $V_{1}, V_{2}$ by analogy with $V_{0}$ ), this appears to be the only course of action to take.

The intertwining relation (54) for $W_{3}$ is now trivial, since

$$
\begin{aligned}
W_{3}\left(V_{1}\left(\phi_{1}, w_{1}\right) \chi_{0}, z\right) & V_{1}(\psi, \zeta) V_{1}\left(\phi_{2}, w_{2}\right) \chi_{0} \\
= & W_{3}\left(V_{1}\left(\phi_{1}, w_{1}\right) \chi_{0}, z\right) V_{1}\left(V_{0}\left(\psi, \zeta-w_{2}\right) \phi_{2}, w_{2}\right) \chi_{0} \\
& \text { by the representation property for } \mathcal{H}_{1}, \\
= & V_{2}\left(V_{0}\left(\psi, \zeta-w_{2}\right) \phi_{2}, w_{2}\right) V_{2}\left(\phi_{1}, w_{1}+z\right) F_{\chi_{0}}(z) \\
= & V_{2}(\psi, \zeta) V_{2}\left(\phi_{2}, w_{2}\right) V_{2}\left(\phi_{1}, w_{1}+z\right) F_{\chi_{0}}(z) \\
& \text { by the representation property for } \mathcal{H}_{2}, \\
= & V_{2}(\psi, \zeta) W_{3}\left(V_{1}\left(\phi_{1}, w_{1}\right) \chi_{0}, z\right) V_{1}\left(\phi_{2}, w_{2}\right) \chi_{0},
\end{aligned}
$$

and so we deduce that

$$
W_{3}\left(V_{1}\left(\phi_{1}, w_{1}\right) \chi_{0}, z\right) V_{1}(\psi, \zeta)=V_{2}(\psi, \zeta) W_{3}\left(V_{1}\left(\phi_{1}, w_{1}\right) \chi_{0}, z\right),
$$

as required. 


\subsubsection{Evaluation of $F_{\chi_{0}}(z)$}

In order to verify the locality relation (55), i.e.

$$
W_{3}(\chi, z) W_{1}(\rho, \zeta)=W_{3}(\rho, \zeta) W_{1}(\chi, z)
$$

we need only check its action on the vacuum $|0\rangle$, since we have the intertwining relations for both $W_{3}$ and $W_{1}$. Then, by the action of $W_{1}$ on $|0\rangle$, c.f. (46), we see that (129) is equivalent to the "skew-symmetry" relation

$$
e^{z L_{-1}} W_{3}(\chi,-z) \rho=W_{3}(\rho, z) \chi
$$

c.f. (61). Putting $\chi=V_{1}\left(\phi_{1}, w_{1}\right) \chi_{0}$ and $\rho=V_{1}\left(\phi_{2}, w_{2}\right) \chi_{0}$, we see from (126) that this is equivalent to the same relation for $F_{\chi_{0}}(z)$, i.e.

$$
F_{\chi_{0}}(z)=e^{z L_{-1}} F_{\chi_{0}}(-z)
$$

[Note that, if we had chosen the two ground states in the definition of $F_{\chi_{0}}(z) \equiv W_{3}\left(\chi_{0}, z\right) \chi_{0}$ to be distinct, then they would be interchanged between the left and the right hand sides of (131). The choice of coincident ground states has been made for simplicity.] Note that the definition of $W_{1}$ in terms of $V_{1}$ was forced upon us by a skew-symmetry relation. However, in this case, skew-symmetry cannot be used to define $W_{3}$, but merely to give a condition which must be satisfied by the arbitrary quantity appearing in its definition.

Consider the locality relation (56). We see from a discussion mirroring that in [15] that we need only verify the relation

$$
\left\langle\chi_{0}\left|\bar{W}_{3}\left(\bar{\chi}_{0}, z\right) V_{2}(\phi, w) W_{3}\left(\chi_{0}, \zeta\right)\right| \chi_{0}\right\rangle=\left\langle\chi_{0}\left|W_{1}\left(\chi_{0}, \zeta\right) V_{0}(\phi, w) \bar{W}_{1}\left(\bar{\chi}_{0}, z\right)\right| \chi_{0}\right\rangle
$$

where $\phi \in \mathcal{H}_{0}$ is arbitrary (with $\theta=1$ ). This reduces to

$$
\left\langle F_{\chi_{0}}(z)\left|V_{2}(\phi, w)\right| F_{\chi_{0}}(\zeta)\right\rangle=\left\langle G_{\chi_{0}}\left(1 / \zeta^{*}\right)\left|V_{0}(\phi, w)\right| G_{\chi_{0}}\left(1 / z^{*}\right)\right\rangle
$$

where

$$
\begin{aligned}
G_{\chi_{0}}(z) & \equiv W_{1}\left(\chi_{0}, 1 / z^{*}\right)^{\dagger} \chi_{0} \\
& =\sum_{\lambda \in \Lambda} \chi_{0}^{\dagger} \gamma_{\lambda}^{\dagger} \chi_{0} e^{A_{1}\left(1 / z^{*}\right)^{\dagger}}|\lambda\rangle
\end{aligned}
$$

This is analogous to the final relation in the $\mathbf{Z}_{2}$ case, and is discussed in the next section.

We also have the locality relation (57). Now

$$
\begin{array}{rlr}
W_{3}(\chi, z) \bar{W}_{3}(\phi, w) W_{3}\left(\chi_{0}, \alpha\right) \chi_{0} & =W_{3}(\chi, z) W_{1}\left(\chi_{0}, \alpha\right) \bar{W}_{1}(\phi, w) \chi_{0} & \text { by (56) } \\
& =W_{3}\left(\chi_{0}, \alpha\right) W_{1}(\chi, z) \bar{W}_{1}(\phi, w) \chi_{0} & \text { by (55) } \\
& =W_{2}(\chi, z) \bar{W}_{2}(\phi, w) W_{3}\left(\chi_{0}, \alpha\right) \chi_{0} &
\end{array}
$$


by (54), since

$$
W_{1}(\chi, z) \bar{W}_{1}(\phi, w)=V_{1}\left(\bar{W}_{2}(\chi, z-w) \phi, w\right)
$$

and

$$
W_{2}(\chi, z) \bar{W}_{2}(\phi, w)=V_{2}\left(\bar{W}_{2}(\chi, z-w) \phi, w\right),
$$

by duality for $\mathcal{V}(\chi, z)$ (using relations independent of this one!). So, we deduce that (57) is valid when acting on the state $F_{\chi_{0}}(z)$, and hence for all states by the interwining relations.

To conclude, all that remains to do in order to prove that this construction defines a consistent (meromorphic bosonic) conformal field theory is to check (59) and define $F_{\chi_{0}}(z) \in \mathcal{H}_{2}$ such that (131), (58) and (133) hold. The approach which we will adopt is to use (131) and (58) to give restrictions on the definition of $F_{\chi_{0}}(z)$, and then the idea is to use any postulated expression satisfying these to check (133). ((59) may be checked independently of all this.) The remainder of this sub-section is devoted to finding a plausible expression for $F_{\chi_{0}}(z)$, and in the next sub-section, we consider the relations (133) and (59). It should be noted that both the definition of $F_{\chi_{0}}(z)$ and the completion of the consistency check for the conformal field theory in the next sub-section are as yet incomplete.

First, note that in order to check (58), we need only verify

$$
\bar{W}_{2}\left(\chi_{0}, z\right) F_{\chi_{0}}(\zeta)=\bar{W}_{2}\left(\chi_{0}, \zeta\right) F_{\chi_{0}}(z) .
$$

We initially postulate that $W_{3}\left(\chi_{0}, z\right) \chi_{0}$ is of the following form

$$
\begin{aligned}
F_{\chi_{0}}(z) & =z^{-\Delta_{0}} \exp \left\{\sum_{r, s>0} \bar{c}_{-r} \cdot \bar{c}_{-s} z^{r+s} D_{r s}\right\} \chi\left(\chi_{0}\right) \\
& =z^{-\Delta_{0}} e^{D(z)} \chi\left(\chi_{0}\right),
\end{aligned}
$$

where $\chi\left(\chi_{0}\right)$ is a twisted sector ground state of conformal weight $\Delta_{0}$. Note that the relations (131) and (138) provide no restrictions on $\chi\left(\chi_{0}\right)$. It will be determined by the locality relation (133). [The factor $z^{-\Delta_{0}}$ is needed to ensure the correct commutation relation with $L_{0}$. This is also to be expected from the postulated symmetry between $W_{3}$ and $\bar{W}_{3}$, since the factor of $z^{-2 \Delta_{0}}$ in the definition of $\bar{W}_{3}$ would then also lead to an overall factor of $z^{-2 \Delta_{0}}(1 / z)^{-\Delta_{0}}=z^{-\Delta_{0}}$ in that operator.]

Let us assume that

$$
e^{\frac{1}{z} L_{1}} F_{\chi_{0}}(\zeta)=\left(1-\frac{\zeta}{z}\right)^{-2 \Delta_{0}} F_{\chi_{0}}\left(\frac{z \zeta}{z-\zeta}\right)
$$

which must hold if we are to have locality for the full theory (see [23] for a discussion of the Möbius transformation properties of vertex operators corresponding to primary states). Then (138) reduces to invariance under the interchange of $z$ and $\zeta$ of the expression

$$
\bar{W}_{2}\left(\chi_{0}, z\right) F_{\chi_{0}}(\zeta)=(z-\zeta)^{-2 \Delta_{0}} \sum_{\lambda \in \Lambda} e^{A_{2}\left(1 / z^{*}\right)^{\dagger}}\left\langle\bar{\chi}_{0}\left|\gamma_{\lambda}^{\dagger} e^{B_{2}^{-}\left(1 / z^{*}\right)^{\dagger}} F_{\chi_{0}}\left(\frac{z \zeta}{z-\zeta}\right)\right| \lambda\right\rangle
$$




$$
\begin{gathered}
=(z-\zeta)^{-2 \Delta_{0}}\left(\frac{z \zeta}{z-\zeta}\right)^{-\Delta_{0}} \sum_{\lambda \in \Lambda} e^{A_{2}\left(1 / z^{*}\right)^{\dagger}} \bar{\chi}_{0}^{\dagger} \gamma_{\lambda}^{\dagger} \chi\left(\chi_{0}\right) \\
\cdot\left\langle\widetilde{0}\left|e^{B_{2}^{-}\left(1 / z^{*}\right)^{\dagger}} e^{D(z \zeta /(z-\zeta))}\right| \widetilde{0}\right\rangle|\lambda\rangle,
\end{gathered}
$$

where $|\widetilde{0}\rangle$ denotes a "vacuum" state in $\mathcal{H}_{2}$, i.e. $\langle\widetilde{0} \mid \widetilde{0}\rangle=1, \bar{c}_{t}|\widetilde{0}\rangle=0$ for $t>0$. Since $\Delta_{0}$ is a multiple of 4 (for $c$ a multiple of 72 ), $(z-\zeta)^{-\Delta_{0}}$ is clearly invariant under the interchange. So, we are left with the expression

$$
e^{A_{2}\left(1 / z^{*}\right)^{\dagger}}\left\langle\widetilde{0}\left|e^{B_{2}^{-}\left(1 / z^{*}\right)^{\dagger}} e^{D(z \zeta /(z-\zeta))}\right| \widetilde{0}\right\rangle|\lambda\rangle
$$

Now, an obvious solution to the skew-symmetry relation (131) is to take

$$
F_{\chi_{0}}(z)=z^{-\Delta_{0}} e^{\frac{1}{2} z L_{-1}} \chi\left(\chi_{0}\right)
$$

However, this is easily seen not to satisfy (140). This observation curtails a naive hope that invariance of (142) under $z \leftrightarrow \zeta$ (under which the argument of $D$ changes sign) could be deduced simply as a consequence of the relation (131), which details the effect of such a sign change.

The matrix element $\langle\widetilde{0}|\ldots| \widetilde{0}\rangle$ gives

$$
\exp \left\{\sum_{\substack{m, n \geq 0 \\ r, s>0}} r B_{m r}^{-}\left(\frac{1}{z}\right) s B_{n s}^{-}\left(\frac{1}{z}\right)\left(\frac{z \zeta}{z-\zeta}\right)^{r+s} D_{r s} b_{-m} \cdot \bar{b}_{-n}\right\} .
$$

So, (142) becomes

$$
\exp \left\{\sum_{m, n \geq 0} P_{m n}(\zeta, z) b_{-m} \cdot \bar{b}_{-n}\right\}
$$

where

$$
P_{m n}(\zeta, z)=\sum_{r, s>0} D_{r s}\left(\begin{array}{c}
r \\
m
\end{array}\right)\left(\begin{array}{l}
s \\
n
\end{array}\right)\left(\frac{\zeta}{\zeta-z}\right)^{r+s}(-z)^{m+n}+A_{m n}^{1}\left(\frac{1}{z}\right) .
$$

Hence we require invariance under the interchange $z \leftrightarrow \zeta$ of the expression

$$
\begin{aligned}
P(x, y, \zeta, z) & \equiv \sum_{m, n \geq 0} P_{m n}(\zeta, z) x^{m} y^{n} \\
& =\sum_{m, n \geq 0} A_{m n}^{1}\left(\frac{1}{z}\right) x^{m} y^{n}+\sum_{r, s>0} D_{r s}\left(\frac{(1-z x) \zeta}{\zeta-z}\right)^{r}\left(\frac{(1-z y) \zeta}{\zeta-z}\right)^{s} .
\end{aligned}
$$

We have an expression for the first of these two sums from (85). Denote the second sum by $g\left(\frac{(1-z x) \zeta}{\zeta-z}, \frac{(1-z y) \zeta}{\zeta-z}\right)$. 
Then we require

$$
g(a, b)-g(1-a, 1-b)=-(1-\omega) \ln \left(\frac{(1-a)^{\frac{1}{3}}-\omega(1-b)^{\frac{1}{3}}}{(-a)^{\frac{1}{3}}-\omega(-b)^{\frac{1}{3}}}\right)+(\omega \rightarrow \bar{\omega}) .
$$

Now consider the constraint given by the skew-symmetry relation (131) on $F_{\chi_{0}}(z)$. In order to consider this, we require a Fock space representation of $e^{z L_{-1}}$. This is derived in Appendix B. Set $d_{r}=\bar{c}_{r} / \sqrt{|r|}$ and $e_{s}=\bar{c}_{s} / \sqrt{|s|}$. Then we have, in matrix notation,

$$
e^{z L_{-1}}=e^{d^{\dagger} \bar{A}(z) e^{\dagger}}: e^{d^{\dagger} \bar{B}(z) d}: \quad: e^{e^{\dagger} \bar{C}(z) e}:
$$

where

$$
\begin{gathered}
\bar{A}_{r s}(z) \equiv \sqrt{r s} A_{r s} z^{r+s} \\
\bar{B}_{r r^{\prime}}(z) \equiv \sqrt{r r^{\prime}} B_{r r^{\prime}} z^{r-r^{\prime}} \\
\bar{C}_{s s^{\prime}}(z) \equiv \sqrt{s s^{\prime}} C_{s s^{\prime}} z^{s-s^{\prime}} .
\end{gathered}
$$

Using the simple normal ordering relation

$$
: e^{\alpha^{\dagger} G \alpha}: e^{\beta \alpha^{\dagger}}=e^{\alpha^{\dagger}(1+G) \beta}: e^{\alpha^{\dagger} G \alpha}:
$$

where $\alpha_{n}$ are bosonic creation and annihilation operators with commutation relations normalised to unity (c.f. the $d$ - and e-oscillators above), we see that

$$
e^{z L_{-1}} F_{\chi_{0}}(-z)=z^{-\Delta_{0}} e^{d^{\dagger} \bar{A}(z) e^{\dagger}}: e^{d^{\dagger} \bar{B}(z) d}: \quad: e^{e^{\dagger} \bar{C}(z) e}: e^{d^{\dagger} \bar{D}(z) e^{\dagger}} \chi\left(\chi_{0}\right),
$$

where

$$
\bar{D}_{r s}(z) \equiv \sqrt{r s} D_{r s}(-z)^{r+s}
$$

becomes

$$
z^{-\Delta_{0}} e^{d^{\dagger} \bar{A}(z) e^{\dagger}} e^{d^{\dagger}(1+\bar{B}(z)) \bar{D}(z)(1+\bar{C}(z))^{T} e^{\dagger}} \chi\left(\chi_{0}\right) .
$$

Therefore, (131) is satisfied if this is equal to

$$
F_{\chi_{0}}(z) \equiv z^{-\Delta_{0}} e^{d^{\dagger} \bar{D}(-z) e^{\dagger}} \chi\left(\chi_{0}\right)
$$

We replace $d_{-r}$ by $x^{-r} / \sqrt{r}$ and $e_{-s}$ by $y^{-s} / \sqrt{s}$ for $r, s>0$ in (156) and (157), and the problem then reduces to verifying the equality of the generating functions for the coefficients of the oscillators in the exponentials.

From (157), we obtain the generating function

$$
\begin{aligned}
f(x, y, z) & =\sum_{r, s>0} x^{-r} y^{-s} D_{r s} z^{r+s} \\
& \equiv g\left(\frac{z}{x}, \frac{z}{y}\right) .
\end{aligned}
$$


The generating function which arises from the second term of (156) is given by

$$
\begin{aligned}
h(x, y, z)= & \sum_{\substack{r, s>0 \\
r^{\prime}, s^{\prime}>0}} \frac{x^{-r} y^{-s}}{\sqrt{r s}}\left(\delta_{r r^{\prime}}+\bar{B}_{r r^{\prime}}(z)\right)\left(\delta_{s s^{\prime}}+\bar{C}_{s s^{\prime}}(z)\right) \sqrt{r^{\prime} s^{\prime}} D_{r^{\prime} s^{\prime}}(-z)^{r^{\prime}+s^{\prime}} \\
= & \sum_{\substack{r^{\prime}, s^{\prime}>0 \\
r>r^{\prime}, s>s^{\prime}}} \frac{x^{-r} y^{-s}}{\sqrt{r s}} \sqrt{r^{\prime} s^{\prime}}\left(\delta_{r r^{\prime}}+\sqrt{r r^{\prime}} \frac{(-z)^{r-r^{\prime}}}{r^{\prime}}\left(\begin{array}{c}
-r^{\prime} \\
r-r^{\prime}
\end{array}\right)\right) \\
& \cdot\left(\delta_{s s^{\prime}}+\sqrt{s s^{\prime}} \frac{(-z)^{s-s^{\prime}}}{s^{\prime}}\left(\begin{array}{c}
-s^{\prime} \\
s-s^{\prime}
\end{array}\right)\right) D_{r^{\prime} s^{\prime}}(-z)^{r^{\prime}+s^{\prime}} \\
= & \sum_{\substack{r^{\prime}, s^{\prime}>0 \\
n, m \geq 0}} x^{-m-r^{\prime}} y^{-n-s^{\prime}} z^{m+n}(-1)^{m+n}\left(\begin{array}{c}
-r^{\prime} \\
m
\end{array}\right)\left(\begin{array}{c}
-s^{\prime} \\
n
\end{array}\right) D_{r^{\prime} s^{\prime}}(-z)^{r^{\prime}+s^{\prime}} \\
= & \sum_{\substack{r^{\prime}, s^{\prime}>0 \\
\equiv}} D_{r^{\prime} s^{\prime}}\left(\frac{z}{x-z}\right)^{r^{\prime}}\left(\frac{z}{y-z}\right)^{s^{\prime}} \\
\equiv & g\left(\frac{z}{z-x}, \frac{z}{z-y}\right) .
\end{aligned}
$$

The first term of (156) contributes

$$
k(x, y, z) \equiv \sum_{r, s>0} x^{-r} y^{-s} A_{r s} z^{r+s} .
$$

So, (131) is satisfied if

$$
g\left(\frac{z}{x}, \frac{z}{y}\right) \equiv g\left(\frac{z}{z-x}, \frac{z}{z-y}\right)+k(x, y, z) .
$$

Using (212), we can easily check that

$$
k(x, y, z)=-(1-\omega) \ln \left(\frac{(1-y / z)^{\frac{1}{3}}-\omega(1-x / z)^{\frac{1}{3}}}{(-y / z)^{\frac{1}{3}}-\omega(-x / z)^{\frac{1}{3}}}\right)+(\omega \rightarrow \bar{\omega}),
$$

and hence skew-symmetry is equivalent to

$$
\begin{gathered}
g(a, b)-g\left(\frac{a}{a-1}, \frac{b}{b-1}\right)=-(1-\omega) \ln \left(\frac{(1-1 / b)^{\frac{1}{3}}-\omega(1-1 / a)^{\frac{1}{3}}}{(1 / b)^{\frac{1}{3}}-\omega(1 / a)^{\frac{1}{3}}}\right) \\
+(\omega \rightarrow \bar{\omega}) .
\end{gathered}
$$

Now, it is easily verified that the relation (140) is equivalent to

$$
L_{1} P_{n}=\left(n-1+\Delta_{0}\right) P_{n-1},
$$


where

$$
F_{\chi_{0}}(z)=z^{-\Delta_{0}} \sum_{n \geq 0} P_{n} z^{n}
$$

and hence to

$$
(r+s) D_{r s}=(r+1) D_{r+1 s}+(s+1) D_{r s+1}+\frac{2}{9} D_{\frac{1}{3} s} D_{r \frac{2}{3}} .
$$

Note that this set of relations is sufficient to determine the coefficients $D_{r s}$ at level $n$ (i.e. $r+s=n$ ) in terms of those at level $n-1$ together with one indeterminate. This single unknown at each level could be provided by consideration of (148) and (163) for $a=b$ to produce an expression for $g(a, a)$. However, we note that in our argument of Appendix A the function

$$
\left.h(a) \equiv \frac{d}{d a} \frac{d}{d b} g(a, b)\right|_{a=b}
$$

appeared in the expression for a particular correlation function, and so we feel that this is a more natural object to consider, not least because it removes the logarithms from (148) and (163), which give

$$
h(a)-h(1-a)=\frac{2 a-1}{9 a^{2}(1-a)^{2}}
$$

and

$$
h(a)-\frac{1}{(1-a)^{4}} h\left(\frac{a}{a-1}\right)=\frac{2-a}{9 a(1-a)^{2}} .
$$

Noting the obvious solution to these, we write

$$
h(a)=\frac{1}{9 a(1-a)^{2}}+k(a)
$$

where

$$
k(a)=k(1-a)=\frac{1}{(1-a)^{4}} k\left(\frac{a}{a-1}\right) .
$$

Now, from $g(a, b) \equiv \sum_{r, s>0} D_{r s} a^{r} b^{s}$, we see that $h(a)$ has a simple pole at the origin with residue $\frac{2}{9} D_{\frac{1}{3}} \frac{2}{3}$. But the skew-symmetry relation (131) implies that $D_{\frac{1}{3} \frac{2}{3}}=\frac{1}{2}$, and so we see from (170) that $k(a)$ is regular at 0 . From (171), $k(a)$ is regular also at 1 and $\infty$, and since these are the only possible poles if our conformal field theory is to be consistent (using the fact that $h(a)$ is essentially a correlation function), we see, from Liouville's theorem, that $k(a)$ is constant. Further, (171) shows us that this constant value is zero.

Now we may use the relations (166) to evaluate the coefficients $D_{r s}$ at successive levels. Carrying through this calculation for the first few levels, we arrive at the (surprisingly asymmetric) conjecture

$$
D_{r s}=\frac{(-1)^{r+s}}{r+s}\left(\frac{1}{3 s}-1\right)\left(\begin{array}{c}
-\frac{2}{3} \\
r-\frac{1}{3}
\end{array}\right)\left(\begin{array}{c}
-\frac{1}{3} \\
s-\frac{2}{3}
\end{array}\right) \text {. }
$$


Note that these coefficients have been independently derived in [21] by rather different techniques. We must now check that this satisfies (166), (148) and (163). The relation (166) is trivially verified. Evaluating $g(a, b)$ directly however is difficult. Let us therefore consider instead the derivative with respect to $b$ of the other two relations. We have

$$
\begin{aligned}
\mu(a, b) \equiv \frac{d}{d b} g(a, b) & =-a^{-\frac{1}{3}} b^{\frac{1}{3}} \frac{d}{d b} \sum_{r, s>0} b^{r} a^{s} \frac{(-1)^{r+s}}{r+s}\left(\begin{array}{c}
-\frac{1}{3} \\
r-\frac{1}{3}
\end{array}\right)\left(\begin{array}{c}
-\frac{2}{3} \\
s-\frac{2}{3}
\end{array}\right) \\
& =\frac{\left(\frac{a}{b}\right)^{\frac{1}{3}}-\left(\frac{a(a-1)}{b(b-1)}\right)^{\frac{1}{3}}}{a-b} .
\end{aligned}
$$

We may then easily check that this satisfies the $b$ derivative of (148) and (163), hence verifying these relations up to addition of an arbitrary function of $a$. Noting that both sides of (163) vanish at $b=0$ shows that this arbitrary function vanishes identically in this case. However, we may have a non-zero anomaly in (148). Since we have already noted that direct calculation of $g(a, b)$ seems difficult, let us consider

$$
\begin{aligned}
\rho(a, b) \equiv \frac{d}{d a} g(a, b)= & \sum_{r, s>0} \frac{a^{r-1} b^{s}}{3 s}(-1)^{r+s}\left(\begin{array}{c}
-\frac{2}{3} \\
r-\frac{1}{3}
\end{array}\right)\left(\begin{array}{c}
-\frac{1}{3} \\
s-\frac{2}{3}
\end{array}\right) \\
& -a^{-\frac{1}{3}} \frac{d}{d a} \sum_{r, s>0} \frac{(-1)^{r+s}}{r+s} a^{r+\frac{1}{3}} b^{s}\left(\begin{array}{c}
-\frac{2}{3} \\
r-\frac{1}{3}
\end{array}\right)\left(\begin{array}{c}
-\frac{1}{3} \\
s-\frac{2}{3}
\end{array}\right) .
\end{aligned}
$$

We may evaluate the second term using (162). Substituting this into the derivative of (148) we obtain the appropriate term on the right hand side. However, we also have a contribution from the first term of equation (174). The $b$ derivative of this term is $-b^{-\frac{1}{3}}(1-b)^{-\frac{1}{3}} a^{-\frac{2}{3}}(1-a)^{-\frac{2}{3}} / 3$, and so the anomalous piece on the right hand side of (148) is

$$
f(a)=-\frac{1}{3} \int_{0}^{1} \frac{d b}{b^{\frac{1}{3}}(1-b)^{\frac{1}{3}}} \int_{\frac{1}{2}}^{a} \frac{d x}{x^{\frac{2}{3}}(1-x)^{\frac{2}{3}}},
$$

(noting that $f\left(\frac{1}{2}\right)=0$ by considering (148) for $a=b=\frac{1}{2}$ ).

We must now adjust our definition (139) of $F_{\chi_{0}}(z)$ in order to compensate for this, without affecting either (163) or (164). Let us therefore try

$$
\begin{aligned}
F_{\chi_{0}}(z) & =z^{-\Delta_{0}} \sum_{\alpha \in \Gamma} \exp \left\{\sum_{r>0} \frac{\bar{\alpha} \cdot \bar{c}_{-r}}{\sqrt{3}} F_{r} z^{r}\right\} \exp \left\{\sum_{r, s>0} \bar{c}_{-r} \cdot \bar{c}_{-s} D_{r, s} z^{r+s}\right\} \chi_{\alpha}\left(\chi_{0}\right) \\
& \equiv z^{-\Delta_{0}} \sum_{\alpha \in \Gamma} e^{F_{\alpha}(z)} e^{D(z)} \chi_{\alpha}\left(\chi_{0}\right),
\end{aligned}
$$

where $\chi_{\alpha}\left(\chi_{0}\right)$ is a twisted sector ground state and $\Gamma$ is an Eisenstein lattice containing $\Lambda$ (and the $\sqrt{3}$ arises naturally from the definition of the action of $b_{0}$ on $|\lambda\rangle$ ). Equation (164) then determines the coefficients

$$
F_{r}=\frac{F}{r}\left(\begin{array}{c}
-\frac{2}{3} \\
r-\frac{1}{3}
\end{array}\right)(-1)^{r-\frac{1}{3}},
$$


for some constant $F$. To check that this does not affect skew-symmetry, we need to go through a similar argument to that leading up to (163). The $a$ derivative of the contribution of (177) to the generating function is clearly $\hat{g}(a)=a^{-\frac{2}{3}}(1-a)^{-\frac{2}{3}}$. Hence, to avoid contradicting (163), we could have

$$
\hat{g}(a)=-\frac{1}{(1-a)^{2}} \hat{g}\left(\frac{a}{a-1}\right) .
$$

This is clearly not true. However, note that we must take the sum over $\alpha \in \Gamma$ into account. (178) is valid up to a factor of $-\omega$, and so if we can arrange so that only every sixth term in the expansion of the first exponential in (176) actually contributes, then we have the desired result. Since $\Gamma$ is an Eisenstein lattice, then for $\alpha \in \Gamma$ we have $-\alpha, \omega \alpha, \bar{\omega} \alpha \in \Gamma$. So, provided that

$$
\chi_{\alpha}\left(\chi_{0}\right)=\chi_{-\alpha}\left(\chi_{0}\right)=\chi_{\omega \alpha}\left(\chi_{0}\right),
$$

we see that, on summing over the lattice $\Gamma$, all but every sixth term does vanish and so skew-symmetry remains valid. [Note that $\chi_{\alpha}\left(\chi_{0}\right)=\chi_{\omega \alpha}\left(\chi_{0}\right)$ is in general necessary in any case in order that $F_{\chi_{0}}(z)$ be meromorphic.]

Finally, we must check that our modification cancels the anomaly $f(a)$. There are two parts to this. Firstly, we verify that the contribution of the $F_{r}$ 's to the relevant generating function produces the appropriate term. Secondly, we must check that the other contributions from the sum over $\alpha \in \Gamma$ (i.e. for $\alpha \neq \lambda$ ) cause no problems in (141).

So, let us begin by considering the term $\alpha=\lambda$. The additional contribution to the generating function $g(a, b)$ due to our new exponential term is given by

$$
\phi(x, z, \zeta)=\sum_{\substack{r>0 \\
n \geq 0}} F_{r}\left(\frac{z \zeta}{z-\zeta}\right)^{r} \cdot r \cdot \frac{(-z)^{n-r}}{r}\left(\begin{array}{l}
r \\
n
\end{array}\right) x^{n},
$$

from commuting $B_{2}^{-}\left(1 / z^{*}\right)^{\dagger}$ past $e^{F_{\alpha}(z \zeta /(z-\zeta))}$ in the matrix element analogous to (142). Rewriting this in terms of the variable $a=(1-z x) \zeta /(\zeta-z)$ as in (148), we find the additional term

$$
\phi(a)=\int_{0}^{a} \frac{F d b}{(1-b)^{\frac{2}{3} b^{\frac{2}{3}}} .}
$$

So, we can cancel our anomaly $f(a)$ if $\phi(a)-\phi(1-a)+f(a)=0$. We see that this is achieved by setting

$$
F=\frac{1}{6} \int_{0}^{1} \frac{d b}{b^{\frac{1}{3}}(1-b)^{\frac{1}{3}}}=\frac{\Gamma\left(\frac{2}{3}\right)^{2}}{2 \Gamma\left(\frac{1}{3}\right)},
$$

using the well known identity for the beta function.

Now consider the remainder of the contribution of our additional exponential. The $\alpha$-dependent piece of (141) is

$$
\sum_{\alpha \in \Gamma} \bar{\chi}_{0}^{\dagger} \gamma_{\lambda}^{\dagger} \chi_{\alpha}\left(\chi_{0}\right) \exp \left\{\sum_{n \geq 0} \frac{\bar{\alpha} \cdot b_{n}}{\sqrt{3}} \phi_{n}\right\},
$$


where

$$
\phi(a)=\sum_{n \geq 0} \phi_{n} x^{n}
$$

This is

$$
\sum_{\alpha \in \Gamma} \bar{\chi}_{0}^{\dagger} \gamma_{\lambda}^{\dagger} \chi_{\alpha+\lambda}\left(\chi_{0}\right) \exp \left\{\sum_{n \geq 0} \frac{\bar{\alpha} \cdot b_{n}}{\sqrt{3}} \phi_{n}\right\} \exp \left\{\sum_{n \geq 0} \frac{\bar{\lambda} \cdot b_{n}}{\sqrt{3}} \phi_{n}\right\},
$$

where the final term is that which we have used to cancel our anomaly. In order that the remainder of (185) be invariant under the locality transformation $a \mapsto 1-a$, we require

$$
\sum_{\alpha \in \gamma} \bar{\chi}_{0}^{\dagger} \gamma_{\lambda}^{\dagger} \chi_{\alpha+\lambda}\left(\chi_{0}\right) e^{\bar{\alpha} \cdot \phi(a) / \sqrt{3}}
$$

be invariant, abusing our notation slightly and writing $\phi$ as a vector. But

$$
\phi(a)=\phi(1)-\phi(1-a)
$$

from (181). Hence, we must have

$$
\sum_{\alpha \in \Gamma} \bar{\chi}_{0}^{\dagger} \gamma_{\lambda}^{\dagger} \chi_{\alpha+\lambda}\left(\chi_{0}\right) e^{\bar{\alpha} \cdot \lambda \phi(1) / 3} e^{\bar{\alpha} \cdot \phi(a) / \sqrt{3}}=\sum_{\alpha \in \Gamma} \bar{\chi}_{0}^{\dagger} \gamma_{\lambda}^{\dagger} \chi_{-\alpha+\lambda}\left(\chi_{0}\right) e^{-\bar{\alpha} \cdot \phi(a) / \sqrt{3}} .
$$

Now

$$
\phi(1)=F \int_{0}^{1} \frac{d a}{a^{\frac{2}{3}}(1-a)^{\frac{2}{3}}}=\frac{1}{2} \Gamma\left(\frac{1}{3}\right) \Gamma\left(\frac{2}{3}\right)=\frac{\pi}{\sqrt{3}}
$$

using the beta function identity once more, together with (182). Let us try

$$
\chi_{\alpha}\left(\chi_{0}\right)=\eta_{\alpha} \chi\left(\chi_{0}\right)
$$

where $\eta_{\alpha}$ is a scalar. Remembering the restriction (179) on $\chi_{\alpha}$, we try

$$
\eta_{\alpha}=e^{\epsilon \alpha \cdot \bar{\alpha}}
$$

for some (complex) scalar $\epsilon$, and try to set

$$
\eta_{\alpha+\lambda} e^{\frac{\bar{\alpha} \cdot \lambda}{3} \phi(1)}=\eta_{-\alpha+\lambda}
$$

Considering the real part of this gives $\operatorname{Re} \epsilon=-\frac{\pi}{12 \sqrt{3}}$, leaving us with a phase $e^{(\bar{\alpha} \cdot \lambda-\alpha \cdot \bar{\lambda}) \pi / 6 \sqrt{3}}$. [Note that this negative value for $\operatorname{Re} \epsilon$ guarantees convergence of our expression (176) for $F_{\chi_{0}}(z)$, which was not well-defined $a$ priori.]

Now, the overall factor of $\bar{\chi}_{0}^{\dagger} \gamma_{\lambda}^{\dagger} \chi\left(\chi_{0}\right)$ could at best be arranged so that it vanishes except when $\lambda \in(1-\omega) \Lambda \equiv \Lambda_{0}$, or at least some coset of $\Lambda_{0} \in \Lambda$. [Note that for $\Lambda_{0}$ the twisted cocycle symmetry factors vanish and so $\gamma_{\lambda}$ is a multiple of the identity in an irreducible representation (which we have seen that we must have from our earlier 
considerations of self-duality.] Suppose $\lambda_{0} \in \Lambda_{0}$. Say $\lambda_{0}=(1-\omega) \lambda$, with $\lambda \in \Lambda$ and set $\bar{\alpha} \cdot \lambda=m+n \omega$ (assuming $\left.\Gamma \subset \Lambda^{*}\right)$. Then $\bar{\alpha} \cdot \lambda_{0}-\alpha \cdot \bar{\lambda}_{0}=\sqrt{3} i(2 n-m)$, so that our remaining phase to be corrected in (192) is $e^{\pi i(2 n-m) / 6}$. Choosing $\operatorname{Im} \epsilon=\pi / 12$ (and hence $\epsilon=\pi \omega / 6 \sqrt{3})$, we find that we are left with a phase $e^{\pi i(m+n) / 3}$. Now our condition on $\Lambda$, i.e. that $(\lambda, \lambda) \in 3 \mathbf{Z}$ for all $\lambda \in \Lambda$, gives us, assuming $\Gamma=\Lambda, m+n \in 3 \mathbf{Z}$, and hence our residual phase is simply $(-1)^{(m+n) / 3}$. However, specific examples show that $(m+n) / 3$ can be odd.

We do not yet understand what further modifications can be made to the definition of $F_{\chi_{0}}(z)$ to correct for this, though note that, if we do not require a self-dual theory, we may project $\Lambda$ on to a sub-lattice $\Lambda^{\prime}$ such that $\lambda \cdot \bar{\lambda} \in 6 \mathbf{Z}$ for all $\lambda \in \Lambda^{\prime}$, for which the locality relation then holds exactly. However, we would expect the construction to work in the self-dual case also, by analogy with the lattice constructions described later. [Note that we can easily insert gamma matrices into the definition of $\chi_{\alpha}\left(\chi_{0}\right)$ which potentially correct the phase. However, as we find when we consider the locality relations $W_{1}(\chi, z) \bar{W}_{2}(\rho, \zeta)=$ $W_{1}(\rho, \zeta) \bar{W}_{2}(\chi, z)$ and $\bar{W}_{3}(\chi, z) W_{3}(\rho, \zeta)=W_{1}(\rho, \zeta) \bar{W}_{1}(\chi, z)$, the optimal solution would seem to be to double the value of $F$ (c.f. (182)), and so we leave this investigation until such matters are fully understood.]

Also note that the expression for $F_{\chi_{0}}(z)$ satisfying the relations which we have examined so far is not unique. In particular, we are free to insert an overall scale (even zero!) into our definition. This scale in particular, and presumably the full expression, will be fixed (at least up to a trivial "gauge" symmetry of the ground states) by a full examination of the locality relation $\bar{W}_{3}(\chi, z) W_{3}(\rho, \zeta)=W_{1}(\rho, \zeta) \bar{W}_{1}(\chi, z)$.

The locality relations $W_{1}(\chi, z) \bar{W}_{2}(\rho, \zeta)=W_{1}(\rho, \zeta) \bar{W}_{2}(\chi, z)$ and $\bar{W}_{3}(\chi, z) W_{3}(\rho, \zeta)=$ $W_{1}(\rho, \zeta) \bar{W}_{1}(\chi, z)$ are the analogues of the final relation in the $\mathbf{Z}_{2}$ case. The approach to their verification follows essentially along the lines set out in 15. Much of this has been done, though the details and one or two problems remain to be completed, and so we leave this to a future publication.

We now look at the possible results of such a construction, and analogies to the links with coding theory discovered in the $\mathbf{Z}_{2}$ case in [16, 14].

\subsection{Results of the Construction for $c=24$}

As noted in section 3.1, an $\mathcal{E}$-lattice has a natural $\mathbf{Z}_{3}$ symmetry, which is inherited by the corresponding $\mathbf{Z}$-lattice $\Lambda_{R} / \alpha$. [The exact form of the converse statement, i.e. that a Z-lattice with a third order NFPA can be written in the form $\Lambda_{R} / \alpha$ for some $\alpha$, where $\Lambda$ is an integral $\mathcal{E}$-lattice, is not immediately apparent, although we shall find it unnecessary, at least in the 24-dimensional case upon which we are focusing, to consider this.] So, we must check which of the Niemeier lattices (the even self-dual lattices in 24 dimensions) admits a third order NFPA. As shown by Venkov 38], each of the lattices is uniquely determined by its set of minimal vectors (those of length squared two), which form the root system of a simply-laced semi-simple Lie algebra. A third order NFPA of a Niemeier lattice must map the minimal vectors into themselves, and so provides a third order NFPA of the root lattice 
of the corresponding semi-simple Lie algebra. Clearly, each component of the algebra (if the algebra is non-simple) must map into itself under the action of the automorphism (otherwise the components must be permuted and we could find a non-zero vector fixed by this action). The third order NFPA's of the root lattices of simple Lie algebras have been classified [32 and only $A_{2}, D_{4}, E_{6}, E_{8}, F_{4}$ and $G_{2}$ admit such automorphisms. (In each case there is only one appropriate conjugacy class in the automorphism group.) So, from the list of possibilities derived by Venkov, we see that the only lattices which could possibly admit a third order NFPA are $E_{8}{ }^{3}, D_{4}{ }^{6}, A_{2}{ }^{12}, E_{6}{ }^{4}$ and $\Lambda_{24}$. Note that, in the case of $E_{8}{ }^{3}$, the lattice $i s$ the root lattice of the Lie algebra, and so it does admit the symmetry. But, in the remaining cases, we must check that the symmetry can be extended to the full lattice.

The twist-invariant subalgebras corresponding to the first four possibilities are $A_{8}{ }^{3}$, $A_{2}{ }^{6}, U(1)^{24}$ and $A_{2}{ }^{12}$ respectively. Since the conformal weight of the twisted sector ground state is $\frac{4}{3}$, then the states of lowest conformal weight coming from the twisted sector are of weight 2, and so there is no enhancement of this (the algebra is generated by the zero modes of the vertex operators corresponding to the states of conformal weight one [23]), i.e. the algebra of the new theory $\widehat{\mathcal{H}}\left(\Lambda_{R} / \alpha\right)$ is just the twist-invariant subalgebra of that of $\mathcal{H}\left(\Lambda_{R} / \alpha\right)$ (which is simply the Lie algebra corresponding to the Niemeier lattice). We prove a theorem in [14] that when the rank of the Lie algebra corresponding to a conformal field theory is equal to the central charge, the conformal field theory is isomorphic to $\mathcal{H}(\Sigma)$ for some even lattice $\Sigma$. Thus, the theories in the first, third and fourth cases must just be $\mathcal{H}\left(A_{8}{ }^{3}\right), \mathcal{H}\left(\Lambda_{24}\right)$ and $\mathcal{H}\left(A_{2}{ }^{12}\right)$ respectively, if they do exist (i.e. if the $\mathbf{Z}_{3}$ construction is consistent). Only $\widehat{\mathcal{H}}\left(D_{4}{ }^{6}\right)$ and $\widehat{\mathcal{H}}\left(\Lambda_{24}\right)$ could provide new theories.

If $D_{4}{ }^{6}$ admits a third order NFPA (and $\widehat{\mathcal{H}}\left(D_{4}{ }^{6}\right)$ is consistent as a conformal field theory), then this would definitely be a new theory, since the algebra $A_{2}{ }^{6}$ does not occur in the list of results in [16]. If the conjecture of FLM, that the self-dual conformal field theory with no states of weight one and with least value of $c$ is unique, is correct, then we must have $\widehat{\mathcal{H}}\left(\Lambda_{24}\right)=V^{\natural}\left(=\widetilde{\mathcal{H}}\left(\Lambda_{24}\right)\right.$, the $\mathbf{Z}_{2}$-twisted orbifold theory), i.e. we would have a new construction of the natural Monster module. We shall discuss this further later. It is hoped that such a construction could throw more light upon the triality structure which, although perhaps rendered a little less mysterious as a result of earlier work [14, 16], we feel still needs to be explained in a more satisfactory setting.

We could check explicitly whether the postulated $\mathbf{Z}_{3}$ symmetries extend to the Niemeier lattices. However, we shall obtain this result naturally from the work of the next subsection in all but the case of the lattice corresponding to $D_{4}{ }^{6}$. Hence, we now merely check whether the third order NFPA of the $D_{4}{ }^{6}$ root lattice extends to the Niemeier lattice $D_{4}{ }^{6}$.

We may describe a Niemeier lattice $\Sigma$ by specifying the appropriate Lie algebra and a set of glue vectors $[8]$. Let $\Sigma_{M}$ be the root lattice of the Lie algebra, i.e. the lattice spanned by the minimal vectors of $\Sigma$. Suppose $\Sigma_{M}=\Sigma_{1} \oplus \ldots \oplus \Sigma_{k}$, where the $\Sigma_{i}$ are the root lattices of the simple components of the Lie algebra. Then any $\lambda \in \Sigma$ must be an element 
of the dual of $\Sigma$ (note that we only require that $\Sigma$ be integral), i.e. $\lambda=\left(\lambda_{1}, \ldots, \lambda_{k}\right)$, where each $\lambda_{i} \in \Sigma_{i}{ }^{*}$. Clearly we need only consider cosets of $\Sigma_{i}$ in $\Sigma_{i}{ }^{*}$. A standard system of representatives of these are referred to as the glue vectors of $\Sigma_{i}$ (usually chosen to be of minimal length). The lattice $\Sigma$ is then generated by $\Sigma_{M}$ together with a set of glue vectors $\left(\lambda_{1}, \ldots, \lambda_{k}\right)$, with each $\lambda_{i}$ a glue vector for $\Sigma_{i}$. We say that the components $\Sigma_{1}, \ldots, \Sigma_{k}$ have been glued together by the glue vectors. (Note that this description can be applied for any integral lattice.)

Let us take as generator matrix for the root lattice of $D_{4}$ the matrix

$$
\left(\begin{array}{llll}
2 & 0 & 0 & 0 \\
1 & 1 & 0 & 0 \\
1 & 0 & 1 & 0 \\
1 & 0 & 0 & 1
\end{array}\right)
$$

Then a set of glue vectors is given by

$$
\begin{array}{cc}
{[0]=(0,0,0,0) ;} & {[1]=\left(\frac{1}{2}, \frac{1}{2}, \frac{1}{2}, \frac{1}{2}\right)} \\
{[2]=(0,0,0,1) ;} & {[3]=\left(\frac{1}{2}, \frac{1}{2}, \frac{1}{2},-\frac{1}{2}\right) .}
\end{array}
$$

The matrix

$$
\left(\begin{array}{cccc}
-1 & 1 & 1 & 1 \\
-1 & -1 & -1 & 1 \\
-1 & 1 & -1 & -1 \\
-1 & -1 & 1 & -1
\end{array}\right)
$$

describes a third order automorphism of this lattice [32].

A set of glue vectors for the Niemeier lattice $D_{4}{ }^{6}$ is given, using the notation of (194) and (195), by [111111] and [0(02332)], where the parentheses include that we should also include all cyclic permutations. Then, in order to check that the symmetry $M$ does not extend to the Niemeier lattice, we need only check that $M$ does not map all of the glue vectors into elements of the lattice. In fact, we must check that no element in the same conjugacy class of the automorphism group of the root lattice of $D_{4}$ as $M$ maps the glue vectors into the Niemeier lattice. Consider $M^{\prime}=h^{-1} M h$, for $h$ an arbitrary element of this automorphism group. Denote by $[n]_{*}$ the coset containing $[n]$ in $D_{4}{ }^{*}$ modulo $D_{4}$. Then, considering the glue corresponding to the first $D_{4}$ component of the Niemeier lattice $D_{4}{ }^{6}$, we see that we must have $M^{\prime}[1]_{*}=[0]_{*}$ or $M^{\prime}[1]_{*}=[1]_{*}$ if $M^{\prime}$ is to extend to the full lattice. Since $[0]_{*}=D_{4}$ and $\left(M^{\prime}\right)^{-1}$ maps $D_{4}$ into itself, we see that we must have the second of the two possibilities, i.e. $M h[1]_{*}=h[1]_{*}$. However, we can easily check that $M[n]_{*}=[n]_{*}$ only for $n=0$. Hence $h[1]_{*}=[0]_{*}$, and acting with $h^{-1}$ on this we find that $h^{-1}[0]_{*}=[1]_{*}$, in contradiction to the fact that $h^{-1} \in \operatorname{Aut}\left(D_{4}\right)$. [Note that in the case of the other Niemeier lattices which potentially admit a third order NFPA, and which we shall show in the next sub-section in fact do, we should in principle consider the possibility that the single conjugacy class in the automorphism group of the corresponding root lattice 
splits into more than one in the automorphism group of the full lattice. However, we have seen that the conformal field theories which are produced must be independent of these potentially distinct $\mathbf{Z}_{3}$ twists, since they are of the form $\mathcal{H}(\Lambda)$, determined solely by the twist-invariant subalgebra arising from the root lattice, at least in the case of all but the Leech lattice, and so we need not consider this situation for our purposes.] Hence $D_{4}{ }^{6}$ does not admit a third order NFPA, and the potentially new theory with algebra $A_{2}{ }^{6}$ does not exist.

What we have shown is that we obtain at most four theories (we still must check that $A_{2}{ }^{12}, \Lambda_{24}$ and $E_{6}{ }^{4}$ do admit third order NFPA's), three of which would be merely theories of the form $\mathcal{H}(\Lambda)$ for $\Lambda$ another Niemeier lattice, while the fourth is most likely to be the Monster module.

\subsection{Straight and Twisted Constructions of $\mathcal{E}$-Lattices from Ternary Codes}

Considering the results of the previous sub-section, we see that the possibilities are as shown in figure 1, where upward-sloping arrows indicate the usual FKS construction and downward-sloping arrows the $\mathbf{Z}_{3}$-twisted construction. This is very reminiscent of the work on binary codes and $\mathbf{Z}_{2}$-twisted conformal field theories, and leads us to strongly suspect both that these results are as shown in the figure (i.e. that the twists lift to the Niemeier lattices and the twisted conformal field theories are consistent) and that there is some code structure to the left of the figure.

However, there appears to be only space for two or perhaps three codes to be inserted. Knowing there are only three self-dual ternary codes of length 12 (two of which contain codewords of weight 12), we are led to consider a construction in 12 dimensions, i.e. to consider a complex construction. Having realised in the previous sections that it is in fact the 12 dimensional $\mathcal{E}$-lattice which is the relevant structure to consider, it now seems natural to attempt to construct $d$-dimensional Eisenstein lattices from length $d$ ternary codes, and then the corresponding $2 d$-dimensional $\mathbf{Z}$-lattices will carry a natural $\mathbf{Z}_{3}$ NFPA.

We shall generalise a pair of constructions due to Sloane [8, 36].

Let $\hat{\mathcal{C}}$ be a self-dual $d$-dimensional ternary code. Define the "straight" construction by

$$
\Lambda_{\mathcal{E}}(\hat{\mathcal{C}})=\hat{\mathcal{C}}+(\omega-\bar{\omega}) \mathcal{E}^{d}
$$

This is clearly an $\mathcal{E}$-lattice (note that $\omega \hat{c}=\hat{c}-(\omega-\bar{\omega}) \bar{\omega} \hat{c}$ for $\hat{c} \in \hat{\mathcal{C}}$, and so we have an Eisenstein module) and further $(\lambda, \lambda) \in 3 \mathbf{Z}$ for $\lambda \in \Lambda_{\mathcal{E}}(\hat{\mathcal{C}})$. From the expression given by Sloane, we see that $\operatorname{det}\left(\Lambda_{\mathcal{E}}(\hat{\mathcal{C}})_{R} / \sqrt{3}\right)=1$, and so $\Lambda_{\mathcal{E}}(\hat{\mathcal{C}})_{R} / \sqrt{3}$ is an even self-dual Zlattice with a third order NFPA induced by the map $\lambda \mapsto \omega \lambda$ on $\Lambda_{\mathcal{E}}(\hat{\mathcal{C}})$. (Note that the dimension of a self-dual ternary code must be a multiple of 4 , and this is consistent with the constraint that the dimension of an even self-dual (Z-)lattice be a multiple of 8 .)

For $\underline{1} \in \hat{\mathcal{C}}(\underline{1}=(1,1, \ldots, 1))$, and hence $d$ a multiple of 12 (say $d=12 D$ ), define the 
lattice $\quad \mathrm{cft}$
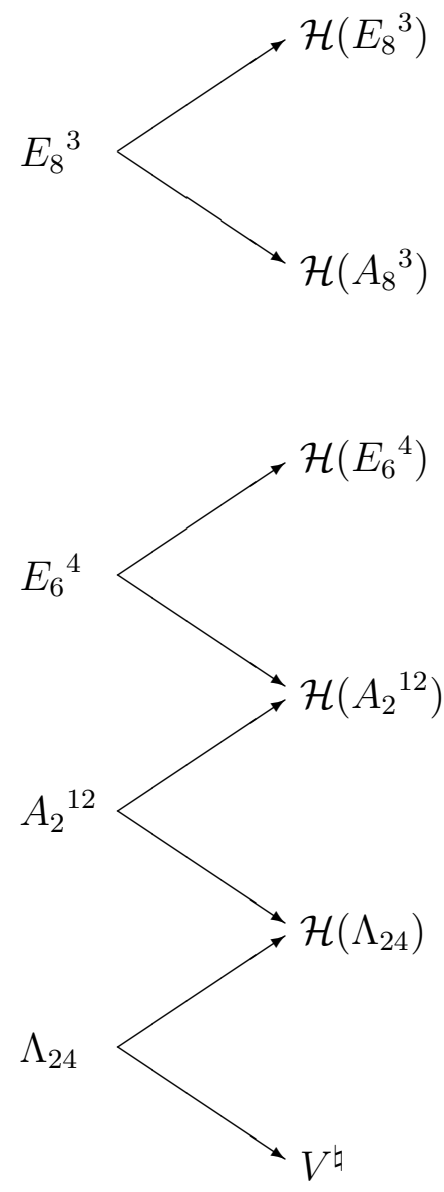

Figure 1:

"twisted" construction by

$$
\tilde{\Lambda}_{\mathcal{E}}(\hat{\mathcal{C}})=\Lambda_{0}(\hat{\mathcal{C}}) \cup \Lambda_{1}(\hat{\mathcal{C}}) \cup \Lambda_{2}(\hat{\mathcal{C}})
$$

where

$$
\begin{gathered}
\Lambda_{0}(\hat{\mathcal{C}})=\hat{\mathcal{C}}+(\omega-\bar{\omega}) \mathcal{E}_{0}^{d} \\
\Lambda_{1}(\hat{\mathcal{C}})=\hat{\mathcal{C}}+(\omega-\bar{\omega})\left(\mathcal{E}_{D}^{d}+\frac{1}{3} \underline{1}\right) \\
\Lambda_{2}(\hat{\mathcal{C}})=\hat{\mathcal{C}}+(\omega-\bar{\omega})\left(\mathcal{E}_{-D}^{d}-\frac{1}{3} \underline{1}\right),
\end{gathered}
$$

with

$$
\mathcal{E}_{\rho}^{d} \equiv\left\{x=\left(x_{1}, \ldots, x_{d}\right) \in \mathcal{E}^{d}: \sum_{i=1}^{d} x_{i} \equiv \rho \bmod (\omega-\bar{\omega})\right\}
$$


We can easily check that this is a lattice. (Note that the requirement that it is an $\mathcal{E}$-module implies, as above, that $(\omega-\bar{\omega}) \bar{\omega} \hat{c} \in(\omega-\bar{\omega}) \mathcal{E}_{0}^{d}$ for $\hat{c} \in \hat{\mathcal{C}}$. This is found to be equivalent to $\underline{1} \cdot \hat{c} \in 3 \mathbf{Z}$, i.e. $\underline{1} \in \hat{\mathcal{C}}^{*}=\hat{\mathcal{C}}$.) We also find that $(\lambda, \lambda) \in 3 \mathbf{Z}$, and $\tilde{\Lambda}_{\mathcal{E}}(\hat{\mathcal{C}})_{R} / \sqrt{3}$ is an even self-dual $2 d$-dimensional $\mathbf{Z}$-lattice with a $\mathbf{Z}_{3}$ NFPA.

[Note that these two constructions are a slightly generalised form of those given in [8]. We have extended the twisted construction to work for $d$ an arbitrary multiple of 12 , rather than just for $d \equiv 12 \bmod 36$, and rescaled our definitions so that the corresponding real lattices are even and self-dual.]

Let us consider the results of these two constructions for $d=12$. Referring to [27], we see that there are three self-dual codes of length 12 , i.e. $\mathcal{C}_{12}, 4 \mathcal{C}_{3}(12)$ and $3 \mathcal{C}_{4} .3 \mathcal{C}_{4}$ does not contain $\underline{1}$, and so cannot induce a twisted construction. Let us identify the corresponding Niemeier lattices.

The vectors of length squared 2 in $\Lambda_{\mathcal{E}}(\hat{\mathcal{C}})_{R} / \sqrt{3}$ correspond to vectors of norm 3 in $\Lambda_{\mathcal{E}}(\hat{\mathcal{C}})$. These are the $27 n_{3}$ vectors of the form

$$
\hat{c}+(\omega-\bar{\omega}) x
$$

with $\hat{c} \in \hat{\mathcal{C}}$ of weight 3 and $x=\left(x_{1}, \ldots, x_{d}\right) \in \mathcal{E}^{d}$ with $x_{i}=\omega \hat{c}_{i},-\bar{\omega} \hat{c}_{i}$ or 0 , together with the $6 d$ vectors

$$
\pm(\omega-\bar{\omega}) \zeta e_{i}
$$

with $\zeta=1, \omega$ or $\bar{\omega}$ and $e_{i}$ the unit vector containing 1 in the $i$ 'th coordinate. The corresponding vectors in $\tilde{\Lambda}_{\mathcal{E}}(\hat{\mathcal{C}})$ are simply the $9 n_{3}$ vectors of the form (203) with $\sum_{i=1}^{d} x_{i}=$ $0 \bmod (\omega-\bar{\omega})$. Hence, the Coxeter number of the corresponding Niemeier lattice is $9 n_{3} / 8+3$ in the straight case and $3 n_{3} / 8$ in the twisted case. Substituting in the $n_{3}$ values of 0,8 and 24 for $\mathcal{C}_{12}, 4 \mathcal{C}_{3}(12)$ and $3 \mathcal{C}_{4}$ respectively, we see that we can extend figure 1 to produce the structure shown in figure 2. (The Coxeter number does not identify the lattices $E_{6}{ }^{4}$ or $E_{8}{ }^{3}$ uniquely (see $\left[8\right.$ ), but clearly $3 \mathcal{C}_{4}$ produces 3 orthogonal components under the straight construction, while we may pick out $E_{6}{ }^{4}$ with equal ease.)

This confirms that we do now indeed have the correct analogy. Note that it also verifies without the need for additional work that the lattices $E_{6}{ }^{4}, A_{2}{ }^{12}$ and $\Lambda_{24}$ do admit an extension of the third order NFPA's of the corresponding root lattices. It is interesting to note that most of the possible $\mathbf{Z}_{3}$ NFPA's of the Niemeier lattices are realised (the

exception being $D_{4}{ }^{6}$ ) and further that all of the ones which are realised exist due to a link with ternary codes.

\subsection{0 "Triality" Structure}

It has been demonstrated by Dong and Mason [17] that $\widehat{\mathcal{H}}\left(\Lambda_{\mathcal{E}}\left(\mathcal{C}_{12}\right)\right)$ is the Monster conformal field theory $V^{\natural}$, by showing essentially that there is a unique irreducible representation of the affine Griess algebra (formed by the modes of the vertex operators corresponding to the states of conformal weight two). However, there should also exist an analogous proof to that in the $\mathbf{Z}_{2}$ case by FLM [20] (and generalised to a wider class of conformal 
code lattice cft
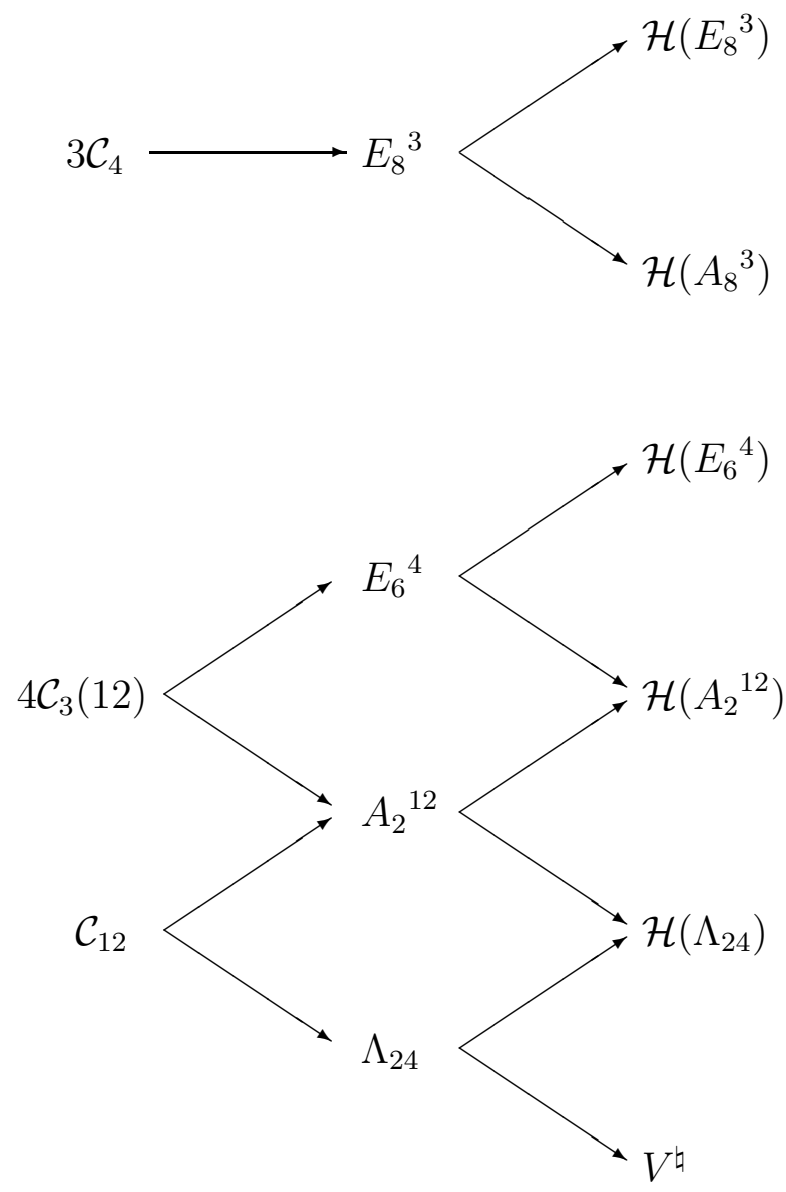

Figure 2:

field theories in [16, 14]). First note that $\mathcal{C}_{12}$, the ternary Golay code, is characterised by properties similar to that of the corresponding binary code, i.e. it is the unique selfdual ternary code of smallest length containing no codewords of weight 3. The complex Leech lattice $\Lambda_{\mathcal{E}}\left(\mathcal{C}_{12}\right)$ has symmetry group $6 S u z$ [8], where $S u z$ is the Suzuki group, one of the finite sporadic simple groups. We note that the non-abelian composition factor in the centraliser of an element in the conjugacy class $3-$ of the Monster is $F_{3-}=S u z$. Comparing this with the results described in [16, 14], i.e. $F_{2-}=C o_{1}=\operatorname{Aut}\left(\Lambda_{24}\right) / \mathbf{Z}_{2}$, suggests that the automorphism group of this conformal field theory is again the Monster. An analogous analysis to that given for the construction of the triality operator in 116, 14 should complete the Suzuki group to the Monster, and also generalise to codes other than $\mathcal{C}_{12}$. At the heart of that analysis lay the affine $s u(2)^{24}$ algebra, while in this case we see that we have an affine $s u(3)^{12}$ algebra, generated by the modes of the vertex operators 
corresponding to the weight one states

$$
a_{-1}^{i}|0\rangle, \quad \bar{a}_{-1}^{i}|0\rangle, \quad| \pm(\omega-\bar{\omega}) \zeta\rangle
$$

where $\zeta=1, \omega, \bar{\omega}$, for each $i=1, \ldots, 12$. However, this analysis remains to be carried through.

\subsection{Results for Higher Order Twists}

Finally, for the sake of completeness, we look at the possible theories which we may obtain from orbifolding with respect to higher order NFPA's. Concentrate again on the $c=24$ theories. We have $c=(p-1) d$. So $(p-1) \mid 24$, and the only primes $p \geq 3$ possible are $p=3$, $5,7,13$. The simple Lie algebras whose root systems admit such symmetries are $A_{p-1}$ in each case, together with $E_{8}$ in the case $p=5$. Hence, the Niemeier lattices which could possibly admit appropriate NFPA's are as shown in figure 3. (Note that $E_{8}{ }^{3}$ certainly does.) The twist invariant subalgebra is $A_{4}{ }^{6}$ in the case of $E_{8}{ }^{3}$. So, as before, we see that this is not a new theory. The remaining cases produce a self-dual conformal field theory with either no $L_{0}=1$ states (in the case of $\Lambda_{24}$ ) or 24 such states (in the case of $\left.A_{p-1}{ }^{24 /(p-1)}\right)$. The twist-invariant subalgebras in the second case are $U(1)^{24}$, so that these theories are simply $\mathcal{H}\left(\Lambda_{24}\right)$ again. The theories in the first case should be $V^{\natural}$ once more, by the FLM conjecture. Note that we do not know of any constructions of lattices from codes over the field $\mathbf{F}_{p}$ which would complete the structure of figure 3, so that in order to verify explicitly that the Niemeier lattices admit an extension of the $\mathbf{Z}_{p}$ NFPA induced by their corresponding root lattice we should have to do some more work. (It is however known that the Leech lattice does possess such automorphisms.)

\section{Conclusions}

We have defined explicitly and essentially verified the consistency of $\mathbf{Z}_{3}$ NFPA orbifolds of lattice conformal field theories, as well as indicating the corresponding results for higher prime ordered twists.

A link with ternary codes analogous to that of $\mathbf{Z}_{2}$ twists with binary codes has been demonstrated, and it is to be hoped that this will further illuminate the still somewhat mysterious structure of the Monster, as well as generalising aspects of this structure to other conformal field theories constructed in this way from ternary codes other than the ternary Golay code.

The relationship to other work on ternary codes [29] is as yet unclear, though promises to reward further investigation with regard to the completion of the classification problem for the self-dual $c=24$ conformal field theories 31 .

Though patently much of the motivation for this work is as an abstract generalisation of previous work, the physical applications of this construction include, as described in the introduction, "realistic" string models containing twisted bosonic fields. This general mechanism of increasing symmetry by combining sectors is also present in superstring 


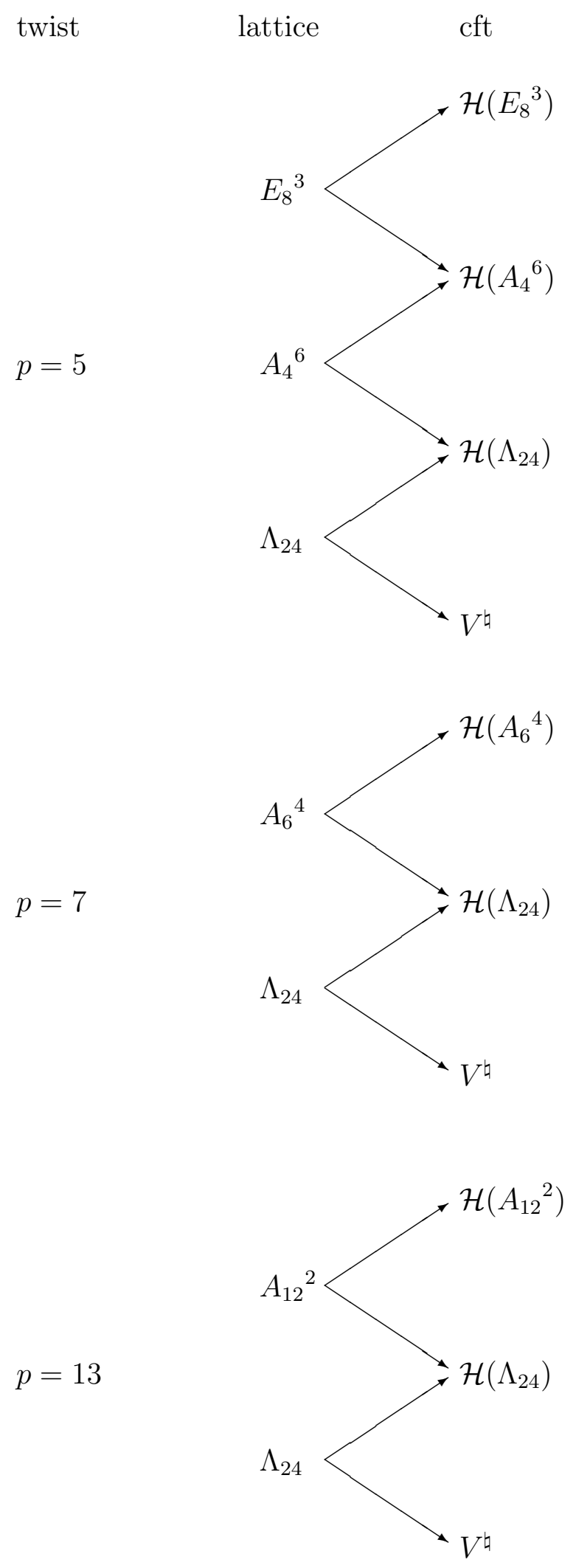

Figure 3: 
models involving twisted world-sheet fermions. Our techniques can clearly be extended to include fermionic fields, and the more explicit results for the structure of the vertex operators than previously found should, in all these models, aid in calculations of, for example, string scattering amplitudes.

Also, the techniques used in the derivation of the implicit form of the twisted sectortwisted sector intertwiner $W_{3}$, which form the essential core of this paper, are exploited in [30] to provide a new perspective on representations of conformal field theories, and perhaps consequently a new approach to other concepts such as fusion could result.

\section{A Twisted Sector Representation of $e^{z L_{-1}}$ for Third Order Twist}

In this appendix we derive an explicitly normal ordered expression for $e^{z L_{-1}}$ in the sector $\mathcal{H}_{2}$ of the $\mathbf{Z}_{3}$-twisted theory.

Write

$$
\begin{gathered}
e^{z L_{-1}}=\exp \left\{\sum_{r, s>0} A_{r s} \bar{c}_{-r} \cdot \bar{c}_{-s} z^{r+s}\right\}: \exp \left\{\sum_{r, r^{\prime}>0} B_{r r^{\prime}} \bar{c}_{-r} \cdot \bar{c}_{r^{\prime}} z^{r-r^{\prime}}\right\} \\
\exp \left\{\sum_{s, s^{\prime}>0} C_{s s^{\prime}} \bar{c}_{-s} \cdot \bar{c}_{s^{\prime}} z^{s-s^{\prime}}\right\}: .
\end{gathered}
$$

Note the absence of a term bilinear in the annihilation operators, since we require $\langle\widetilde{0}| e^{z L_{-1}}=$ 0 . Define

$$
\langle\widetilde{x}, \widetilde{y}| \equiv\langle\widetilde{0}| \sum_{r, s>0} \frac{x^{-r} y^{-s}}{r s} \bar{c}_{r} \cdot \bar{c}_{s}
$$

Set

$$
\begin{aligned}
A(x, y, z) & \equiv\left\langle\widetilde{x}, \widetilde{y}\left|e^{z L_{-1}}\right| \widetilde{0}\right\rangle \\
= & \sum_{r, s>0} A_{r s}\left(\frac{z}{x}\right)^{r}\left(\frac{z}{y}\right)^{s} d,
\end{aligned}
$$

from (206) and (207). But

$$
\begin{aligned}
& \frac{d}{d z} A(x, y, z)=\left\langle\widetilde{x}, \widetilde{y}\left|e^{z L_{-1}} L_{-1}\right| \widetilde{0}\right\rangle \\
& =\left\langle\widetilde{x}, \widetilde{y}\left|e^{z L_{-1}} \bar{c}_{-\frac{1}{3}} \cdot \bar{c}_{-\frac{2}{3}}\right| \widetilde{0}\right\rangle \\
& =\left\langle\widetilde{x}, \widetilde{y}\left|\sum_{n, m \geq 0}(-z)^{n+m}\left(\begin{array}{c}
-\frac{1}{3} \\
n
\end{array}\right)\left(\begin{array}{c}
-\frac{2}{3} \\
m
\end{array}\right) \bar{c}_{-\frac{1}{3}-n} \cdot \bar{c}_{-\frac{2}{3}-m} e^{z L-1}\right| \widetilde{0}\right\rangle \\
& =\langle\widetilde{0}| \sum_{r, s>0} \frac{x^{-r} y^{-s}}{r s} \bar{c}_{r} \cdot \bar{c}_{s} \sum_{n, m \geq 0}(-z)^{n+m}
\end{aligned}
$$




$$
\left(\begin{array}{c}
-\frac{1}{3} \\
n
\end{array}\right)\left(\begin{array}{c}
-\frac{2}{3} \\
m
\end{array}\right) \bar{c}_{-\frac{1}{3}-n} \cdot \bar{c}_{-\frac{2}{3}-m} e^{z L_{-1}}|\widetilde{0}\rangle
$$

Clearly, only the first term in the action of the series expansion of $e^{z L_{-1}}$ on $|\widetilde{0}\rangle$ gives a non-zero contribution to (208), as we require an equal number of creation and annihilation operators. Hence

$$
\begin{aligned}
\frac{d}{d z} A(x, y, z) & =\left\langle\widetilde{0}\left|\sum_{r, s>0} \frac{x^{-r} y^{-s}}{r s} \bar{c}_{r} \cdot \bar{c}_{s} \sum_{n, m \geq 0}(-z)^{n+m}\left(\begin{array}{c}
-\frac{1}{3} \\
n
\end{array}\right)\left(\begin{array}{c}
-\frac{2}{3} \\
m
\end{array}\right) \bar{c}_{-\frac{1}{3}-n} \cdot \bar{c}_{-\frac{2}{3}-m}\right| \widetilde{0}\right\rangle \\
& =\sum_{r, s>0} x^{-r} y^{-s}\left(\begin{array}{c}
-\frac{1}{3} \\
r-\frac{1}{3}
\end{array}\right)\left(\begin{array}{c}
-\frac{2}{3} \\
s-\frac{2}{3}
\end{array}\right)(-z)^{r+s-1} d
\end{aligned}
$$

i.e.

$$
A(z, y, z)=-\sum_{r, s>0}\left(\frac{z}{x}\right)^{r}\left(\frac{z}{y}\right)^{s}\left(\begin{array}{c}
-\frac{1}{3} \\
r-\frac{1}{3}
\end{array}\right)\left(\begin{array}{c}
-\frac{2}{3} \\
s-\frac{2}{3}
\end{array}\right) d \frac{(-z)^{r+s}}{r+s}
$$

(The constant of integration vanishes as $A(x, y, 0)=0$.) Therefore we have, from (208) and (211),

$$
A_{r s}=-\left(\begin{array}{c}
-\frac{1}{3} \\
r-\frac{1}{3}
\end{array}\right)\left(\begin{array}{c}
-\frac{2}{3} \\
s-\frac{2}{3}
\end{array}\right) \frac{(-1)^{r+s}}{r+s}
$$

Similarly, define

$$
B(x, y, z)=\sum_{r, r^{\prime}>0}\left\langle\widetilde{0}\left|\frac{x^{-r}}{r} \bar{c}_{r}^{i} e^{z L_{-1}} \frac{y^{r^{\prime}}}{r^{\prime}} \bar{c}_{-r^{\prime}}^{i}\right| \widetilde{0}\right\rangle,
$$

(with summation over $i=1, \ldots, d$ implied). Then from (206) we have

$$
B(x, y, z)=\sum_{r>0} \frac{1}{r}\left(\frac{y}{x}\right)^{r} d+\sum_{r, r^{\prime}>0}\left\langle\widetilde{0}\left|\frac{x^{-r}}{r} \bar{c}_{r}^{i} B_{r r^{\prime}} \bar{c}_{-r} \cdot \bar{c}_{r^{\prime}} \frac{y^{r^{\prime}}}{r^{\prime}} \bar{c}_{-r^{\prime}}^{i}\right| \widetilde{0}\right\rangle
$$

(the higher order terms in the expansion of the exponentials in (206) giving no contribution as clearly at least two annihilation operators annihilate $c_{-r^{\prime}}^{i}|\widetilde{0}\rangle$, and similarly $\langle\widetilde{0}| c_{r}^{i}$ annihilates at least two creation operators), i.e.

$$
B(x, y, z)=\sum_{r>0} \frac{1}{r}\left(\frac{y}{x}\right)^{r} d+d \sum_{r, r^{\prime}>0} B_{r r^{\prime}}\left(\frac{z}{x}\right)^{r}\left(\frac{y}{z}\right)^{r^{\prime}} .
$$

But

$$
\begin{aligned}
\frac{d}{d z} B(x, y, z) & =\sum_{r, r^{\prime}>0}\left\langle\widetilde{0}\left|\frac{x^{-r}}{r} \bar{c}_{r}^{i} e^{z L_{-1}} L_{-1} \frac{y^{r^{\prime}}}{r^{\prime}} \bar{c}_{-r^{\prime}}^{i}\right| \widetilde{0}\right\rangle \\
& =\sum_{r, r^{\prime}>0}\left\langle\widetilde{0}\left|\frac{x^{-r}}{r} \bar{c}_{r}^{i} e^{z L_{-1}} y^{r^{\prime}} \bar{c}_{-r^{\prime}-1}^{i}\right| \widetilde{0}\right\rangle
\end{aligned}
$$


the contribution from $L_{-1}|\widetilde{0}\rangle$ vanishing by a similar argument again. Thus

$$
\begin{aligned}
\frac{d}{d z} B(x, y, z) & =\sum_{\substack{r, r^{\prime}>0 \\
n \geq 0}}\left\langle\widetilde{0}\left|\frac{x^{-r}}{r} \bar{c}_{r}^{i} y^{r^{\prime}}\left(\begin{array}{c}
-r^{\prime}-1 \\
n
\end{array}\right)(-z)^{n} \bar{c}_{-r^{\prime}-n-1}^{i}\right| \widetilde{0}\right\rangle \\
& =\sum_{r^{\prime}>0}\left(\frac{y}{x}\right)^{r^{\prime}}\left(1-\frac{z}{x}\right)^{-r^{\prime}-1} \frac{1}{x} d .
\end{aligned}
$$

So

$$
B(x, y, z)=\sum_{r^{\prime}>0}\left(\frac{y}{x}\right)^{r^{\prime}}\left(1-\frac{z}{x}\right)^{-r^{\prime}} \frac{1}{r^{\prime}} d,
$$

since $B(x, y, 0)=\sum_{r>0} \frac{1}{r}\left(\frac{y}{x}\right)^{r} d$ from (215). Expanding (218) gives

$$
\begin{aligned}
B(x, y, z) & =\sum_{\substack{r^{\prime} \geq 0 \\
r \geq r^{\prime}}}\left(\frac{y}{x}\right)^{r^{\prime}}\left(-\frac{z}{x}\right)^{r-r^{\prime}}\left(\begin{array}{c}
-r^{\prime} \\
r-r^{\prime}
\end{array}\right) \frac{1}{r^{\prime}} d \\
& =\sum_{\substack{r^{\prime}>0 \\
r \geq r^{\prime}}}\left(\frac{y}{z}\right)^{r^{\prime}}\left(\frac{z}{x}\right)^{r}(-1)^{r-r^{\prime}}\left(\begin{array}{c}
-r^{\prime} \\
r-r^{\prime}
\end{array}\right) \frac{1}{r^{\prime}} d,
\end{aligned}
$$

and comparing with (214) we see that

$$
B_{r r^{\prime}}=\left\{\begin{array}{ll}
(-1)^{r-r^{\prime}}\left(\begin{array}{c}
-r^{\prime} \\
r-r^{\prime}
\end{array}\right) \frac{1}{r^{\prime}} & \text { for } r>r^{\prime} \\
0 & \text { otherwise }
\end{array} .\right.
$$

Similarly

$$
C_{s s^{\prime}}=\left\{\begin{array}{ll}
(-1)^{s-s^{\prime}}\left(\begin{array}{c}
-s^{\prime} \\
s-s^{\prime}
\end{array}\right) \frac{1}{s^{\prime}} & \text { for } s>s^{\prime} \\
0 & \text { otherwise }
\end{array} .\right.
$$

\section{B Symmetry Between the Vertex Operators $W_{3}$ and $\bar{W}_{3}$}

The locality relations (56) and (57) together with the obvious isomorphism between $W_{1}(\rho, \zeta) \bar{W}_{1}(\chi, z)$ and $W_{2}(\bar{\rho}, \zeta) \bar{W}_{2}(\bar{\chi}, z)$ given by interchange of the oscillators $c$ and $\bar{c}$ suggests a corresponding symmetry between the vertex operators $W_{3}(\chi, z)$ and $\bar{W}_{3}(\bar{\chi}, z)$, which would be expected naively in any case. In this appendix, we shall verify this symmetry, making use of the consistency of the $\mathbf{Z}_{3}$-twisted conformal field theory.

For a consistent conformal field theory, $\bar{W}_{3}$ must satisfy a relation analogous to that defining $W_{3}$, i.e. (126). Hence, we need only check the symmetry of $W_{3}\left(\chi_{0}, z\right) \chi_{0} \equiv$ $z^{-\Delta_{0}} F_{\chi_{0}}^{\prime}(z)$ and $\bar{W}_{3}\left(\bar{\chi}_{0}, z\right) \bar{\chi}_{0} \equiv z^{-\Delta_{0}} \bar{F}_{\chi_{0}}^{\prime}(z)$. From (44) we have

$$
\bar{W}_{3}\left(\bar{\chi}_{0}, z\right)=z^{-2 \Delta_{0}} W_{3}^{\dagger}\left(\chi_{0}, 1 / z^{*}\right) .
$$


Let $\psi$ be a state in the untwisted sector (with $\theta=1$ strictly). Then, by the conjugate of (54),

$$
V_{1}(\psi, \zeta) \bar{W}_{3}\left(\bar{\chi}_{0}, z\right)=z^{-2 \Delta_{0}} W_{3}^{\dagger}\left(\chi_{0}, 1 / z^{*}\right) V_{2}(\psi, \zeta)
$$

and hence

$$
\left\langle\chi_{0}\left|V_{1}(\psi, \zeta)\right| \bar{F}_{\chi_{0}}^{\prime}(z)\right\rangle=\left\langle F_{\chi_{0}}^{\prime}\left(1 / z^{*}\right)\left|V_{2}(\psi, \zeta)\right| \bar{\chi}_{0}\right\rangle
$$

Let us consider only the bilinear part of $\bar{F}_{\chi_{0}}^{\prime}(z)$, i.e we take

$$
\bar{W}_{3}\left(\bar{\chi}_{0}, z\right) \bar{\chi}_{0}=z^{-\Delta_{0}} \exp \left\{\sum_{r, s>0} c_{-r} \cdot c_{-s} z^{r+s} \bar{D}_{r s}\right\} \bar{\chi}\left(\chi_{0}\right)
$$

We know that $\bar{W}_{3}$ must satisfy a relation analogous to (140), and therefore to prove coincidence of the generating functions for $D_{r s}$ and $\bar{D}_{r s}$ we need only verify equality for the diagonal forms (c.f. the argument given for the derivation of an expression for the bilinear part of $\left.F_{\chi_{0}}(z)\right)$, i.e. we must show

$$
\bar{g}(x, x)=g(x, x)
$$

where

$$
\bar{g}(x, y) \equiv \sum_{r, s>0} \bar{D}_{r s} x^{r} y^{s}
$$

by analogy with the expression for $g$ given following ((147)).

Consider $\psi=\psi_{L}$ in (224). This gives us

$$
\sum_{r, s>0} r s d \bar{D}_{r s} z^{r+s} \zeta^{-r-s-2}+\frac{d}{9} \frac{1}{\zeta^{2}}=\sum_{r, s>0} r s d D_{r s}{ }^{*} z^{-r-s} \zeta^{r+s-2}+\frac{d}{9} \frac{1}{\zeta^{2}}
$$

i.e., noting that the coefficients $D_{r s}$ which we evaluated in the paper are real,

$$
\left(\frac{z}{\zeta}\right)^{2} \bar{h}\left(\frac{z}{\zeta}\right)=\left(\frac{\zeta}{z}\right)^{2} h\left(\frac{\zeta}{z}\right)
$$

where

$$
\left.h(a) \equiv \frac{d}{d a} \frac{d}{d b} g(a, b)\right|_{a=b}
$$

and similarly for $\bar{h}$.

However, we see from (148) and (163) that $a^{2} h(a)$ is invariant under $a \mapsto 1 / a$, and so $\bar{h}(a)=h(a)$ as required. A similar argument gives the analogous result for the lattice dependent piece of $F_{\chi_{0}}(z)$. 


\section{References}

[1] I. Antoniadis and C. Bachas. 4d fermionic superstrings with arbitrary twists. Nucl. Phys. B298 (1988) 586.

[2] I. Antoniadis, C. Bachas, and C. Kounnas. Four-dimensional superstrings. Nucl. Phys. B289 (1987) 87.

[3] A.A. Belavin, A.M. Polyakov, and A.B. Zamolodchikov. Infinite conformal symmetry in two-dimensional quantum field theory. Nucl. Phys. B241 (1984) 333.

[4] R. Bluhm, L. Dolan, and P. Goddard. A new method of incorporating symmetry into superstring theory. Nucl. Phys. B289 (1987) 364.

[5] R. Bluhm, L. Dolan, and P. Goddard. Unitarity and modular invariance as constraints on four-dimensional superstrings. Nucl. Phys. B309 (1988) 330.

[6] R.E. Borcherds. Vertex algebras, Kac-Moody algebras, and the Monster. Proc. Natl. Acad. Sci. USA 83 (1986) 3068.

[7] J. Cardy. Conformal invariance. In C. Domb and J. Lebowitz, editors, Phase Transitions and Critical Phenomena, page 55. Academic Press, London, 1987.

[8] J.H. Conway and N.J.A. Sloane. Sphere Packings, Lattices and Groups. SpringerVerlag, New York, 1988.

[9] E. Corrigan and T.J. Hollowood. A bosonic representation of the twisted string emission vertex. Nucl. Phys. B303 (1988) 135.

[10] E. Corrigan and T.J. Hollowood. Comments on the algebra of straight, twisted and intertwining vertex operators. Nucl. Phys. B304 (1988) 77.

[11] L. Dixon, D. Friedan, E. Martinec, and S. Shenker. The conformal field theory of orbifolds. Nucl. Phys. B282 (1987) 13.

[12] L. Dixon, J. Harvey, C. Vafa, and E. Witten. Strings on orbifolds. Nucl. Phys. B261 (1985) 678.

[13] L. Dixon, J. Harvey, C. Vafa, and E. Witten. Strings on orbifolds (II). Nucl. Phys. B274 (1986) 285.

[14] L. Dolan, P. Goddard, and P. Montague. Conformal field theories, representations and lattice constructions. Submitted for publication to Communications in Mathematical Physics, hep-th/9410029.

[15] L. Dolan, P. Goddard, and P. Montague. Conformal field theory of twisted vertex operators. Nucl. Phys. B338 (1990) 529. 
[16] L. Dolan, P. Goddard, and P. Montague. Conformal field theory, triality and the Monster group. Phys. Lett. B236 (1990) 165.

[17] C. Dong and G. Mason. On the construction of the moonshine module as a $\mathbf{Z}_{p}$-orbifold. Santa Cruz preprint, 1992.

[18] I. Frenkel, J. Lepowsky, and A. Meurman. A natural representation of the FischerGriess Monster with the modular function $J$ as character. Proc. Natl. Acad. Sci. USA 81 (1984) 3256.

[19] I. Frenkel, J. Lepowsky, and A. Meurman. A moonshine module for the Monster. In J. Lepowsky et al., editors, Vertex Operators in Mathematics and Physics, Proc. 1983 MSRI Conference, page 231, New York, 1985. Springer-Verlag.

[20] I. Frenkel, J. Lepowsky, and A. Meurman. Vertex Operator Algebras and the Monster. Academic Press, New York, 1988.

[21] B. Gato. Construction of vertex operators using operator formalism techniques. Nucl. Phys. B322 (1989) 555.

[22] P. Ginsparg. Applied conformal field theory. Preprint HUTP-88/A054, 1988.

[23] P. Goddard. Meromorphic conformal field theory. In Infinite dimensional Lie algebras and Lie groups: Proceedings of the CIRM-Luminy Conference, page 556, Singapore, 1989. World Scientific.

[24] T.J. Hollowood. Twisted Strings, Vertex Operators and Algebras. PhD thesis, Durham University, 1988.

[25] J. Lepowsky. Calculus of twisted vertex operators. Proc. Natl. Acad. Sci. USA 82 (1985) 8295.

[26] J. Lepowsky and R.L. Wilson. The structure of standard modules, I: Universal algebras and the Rogers-Ramanujan identities. Invent. Math. 77 (1984) 199.

[27] C.L. Mallows, V. Pless, and N.J.A. Sloane. Self-dual codes over GF(3). SIAM J. Appl. Math. 31 (1976) 649.

[28] P.S. Montague. Codes, Lattices and Conformal Field Theories. PhD thesis, Cambridge University, 1991.

[29] P.S. Montague. A new construction of lattices from codes over GF(3). Accepted for publication in Discrete Mathematics, 1994.

[30] P.S. Montague. On representations of conformal field theories and the construction of orbifolds. Submitted for publication, 1994.

[31] P.S. Montague. Orbifold constructions and the classification of self-dual c=24 conformal field theories. Nucl. Phys. B428 (1994) 233. hep-th/9403088. 
[32] R.G. Myhill. Automorphisms and Twisted Vertex Operators. PhD thesis, Durham University, 1987.

[33] A.N. Schellekens. Classification of ten-dimensional heterotic strings. Phys. Lett. B277 (1992) 277.

[34] A.N. Schellekens. Meromorphic $c=24$ conformal field theories. CERN-TH.6478/92, 1992.

[35] A.N. Schellekens. Seventy relatives of the Monster module. NIKHEF-H/93-08, 1993.

[36] N.J.A. Sloane. Self-dual codes and lattices. In Relations Between Combinatorics and Other Parts of Mathematics: Proceedings of Symposia in Pure Mathematics, volume 34, page 272, Providence, R.I., 1979. American Mathematical Society.

[37] M.P. Tuite. Monstrous moonshine from orbifolds. CMP 146 (1992) 277.

[38] B.B. Venkov. The classification of the integral even unimodular 24-dimensional quadratic forms. Proceedings of the Steklov Institute of Mathematics 4 (1980) 63. 